\title{
On Uniform Conditions for the Existence of Mixed Strategy Equilibria
}

\section{Pavlo Prokopovych}

Kyiv School of Economics and Kyiv Economics Institute

Nicholas C. Yannelis

University of Iowa

The University of Manchester 


\title{
On Uniform Conditions for the Existence of Mixed Strategy Equilibria
}

\author{
Pavlo Prokopovych ${ }^{1}$ and Nicholas C. Yannelis ${ }^{2}$
}

\begin{abstract}
Embarking from the concept of uniform payoff security (Monteiro P.K., Page F.H, J Econ Theory 134: 566-575, 2007), we introduce two other uniform conditions and then study the existence of mixed strategy Nash equilibria in games where the sum of the payoff functions is not necessarily upper semicontinuous.
\end{abstract}

Keywords Discontinuous game; Diagonally transfer continuous game; Payoff secure game; Mixed strategy equilibrium; Transfer lower semicontinuity

JEL classification Numbers C65; C72

Date $\quad$ March 15, 2012

\footnotetext{
${ }^{1}$ Pavlo Prokopovych, Kyiv School of Economics, 13 Yakira, 3d floor, Kyiv 04119, Ukraine, Email: pprokopo@gmail.com

${ }^{2}$ Nicholas C. Yannelis, Department of Economics, Tippie College of Business, University of Iowa, Iowa City, IA 52242-1994, USA; Economics - School of Social Sciences, The University of Manchester, Oxford Road, Manchester M13 9PL, UK, E-mail: nicholasyannelis@gmail.com
} 


\section{Introduction}

In this paper we study the existence of mixed strategy equilibria in compact Borel games in which the sum of the payoff functions is not necessarily upper semicontinuous. The existence of a mixed strategy Nash equilibrium in a game is often shown by invoking Reny's equilibrium existence theorem (Reny 1999), according to which a compact Borel game has a mixed strategy Nash equilibrium if its mixed extension is better-reply secure. ${ }^{3}$ In its turn, better-reply security is implied by two conditions: reciprocal (or weak reciprocal) upper semicontinuity and payoff security. As was shown by Simon (1987, Example 5), not every reciprocally upper semicontinuous game has a reciprocally upper semicontinuous mixed extension. Consequently, imposing the assumption that the mixed extension of a game is reciprocally upper semicontinuous is pretty close to assuming explicitly that the sum of the payoff functions is upper semicontinuous. Dasgupta and Maskin (1986, Theorem 5b) provided a set of sufficient conditions for the existence of a mixed strategy Nash equilibrium in a subclass of reciprocally upper semicontinuous games.

Establishing the payoff security of the mixed extension of a game is also often quite challenging. The concept of uniform payoff security, introduced by Monteiro and Page (2007), makes the problem considerably more tractable in games where it is applicable, including catalog games (Page and Monteiro 2003) and voting models (Carbonell-Nicolau and Ok 2007). In this paper two modifications of the uniform payoff security condition are proposed to broaden the class of games in which the existence of mixed strategy Nash equilibria follows from a uniform, easily tractable condition

First we consider games with diagonally transfer continuous mixed extensions. Baye, Tian, and Zhou (1993) showed that the existence of a pure strategy Nash equilibrium in diagonally transfer continuous games follows from a generalization of the Knuster-Kuratowski-Mazurkiewicz (KKM) lemma. In Section 2 of this paper, the Ky Fan minimax inequality, in a slightly generalized form, is used to prove that every compact Borel game whose

\footnotetext{
${ }^{3} \mathrm{~A}$ number of results generalizing Reny's equilibrium existence theorem have been obtained recently (see, e.g., Barelli and Soza 2009; Bich 2009; Carmona 2011; de Castro 2011; McLennan, Monteiro, and Tourky 2011)
} 
mixed extension is diagonally transfer continuous has a mixed strategy Nash equilibrium. In particular, the mixed extension of a game is diagonally transfer continuous if the conventional assumptions hold: the extension is payoff secure and the game is upper semicontinuous-sum. Then, by introducing uniform diagonal security in Section 3, we extend the concept of uniform payoff security to diagonally transfer continuous games. In upper semicontinuoussum games, these two uniform conditions coincide. However, if a compact Borel game is uniformly diagonally secure, it has a mixed strategy Nash equilibrium, which makes it possible to avoid verifying the reciprocal upper semicontinuity of the game's mixed extension.

Example 1 is a slight modification of the Tullock rent-seeking game in which it is additionally assumed that the favor the players vie for may be granted to a third party with probability one-half if at least one player exerts no effort at all. Notwithstanding the fact that the game is not better-reply secure, it is not only diagonally transfer continuous, it is also uniformly diagonally secure; that is, the game has a mixed strategy Nash equilibrium.

In Section 4 we introduce another uniform condition, called uniform hospitality. The notion of a hospitable game is based on the notion of a hospitable strategy, introduced by Duggan (2007). Though uniform hospitality, in a certain sense, is stronger that uniform payoff security (see, e.g., Lemma 6), it might be useful in applications. For instance, all-pay auctions are both uniformly payoff secure and uniformly hospitable. At the same time, if, in a proof, some payoffs are to be modified, uniform hospitality tends to be used side by side with uniform payoff security.

In Section 5 we study the existence of mixed strategy equilibria in two player games on the unit square. The focus is on Theorem $5 \mathrm{~b}$ of Dasgupta and Maskin (1986). Bagh (2010) showed with the aid of examples that the hypotheses of Theorem 5b are not in full harmony with its proof and put forward a modification of the theorem covering mostly Bertrand-Edgeworth games. The games covered by the modification of Theorem $5 \mathrm{~b}$ presented in Section 5 (Theorem 3) possess the following two properties: (a) they are uniformly payoff secure; (b) if a player's payoff is to be modified at a discontinuity point $(z, z)$, the player's payoff function is strongly uniformly hospitable at $z$. As an application of Theorem 3, a probabilistic spatial voting model from Ball (1999) is considered. 


\section{The Model and Some Facts}

We consider a game $G=\left(X_{i}, u_{i}\right)_{i \in I}$, where $I=\{1, \ldots, n\}$, each player $i$ 's pure strategy set $X_{i}$ is a nonempty, compact subset of a metrizable topological vector space, and each payoff function $u_{i}$ is a bounded Borel measurable function from the Cartesian product $X=\prod_{i \in I} X_{i}$, equipped with the product topology, to $\mathbb{R}$. Under these conditions, $G=\left(X_{i}, u_{i}\right)_{i \in I}$ is called a compact Borel game. In this paper, by a game we mean a compact Borel game. The following definition of a payoff secure game was given by Reny (1999).

Definition 1 In $G=\left(X_{i}, u_{i}\right)_{i \in I}$, player $i$ can secure a payoff of $\alpha \in \mathbb{R}$ at $x \in X$ if there exists $d_{i} \in X_{i}$ such that $u_{i}\left(d_{i}, x_{-i}^{\prime}\right) \geq \alpha$ for all $x_{-i}^{\prime}$ in some open neighborhood of $x_{-i}$. The game $G$ is payoff secure if, for every $x \in X$ and every $\varepsilon>0$, each player $i$ can secure a payoff of $u_{i}(x)-\varepsilon$ at $x$.

Payoff security can be reformulated in terms of transfer lower semicontinuity, due to Tian (1992).

Definition 2 Let $Z$ and $Y$ be two topological spaces. A function $f: Z \times$ $Y \mapsto \mathbb{R}$ is $\lambda$-transfer lower semicontinuous in $y$ if, for every $(z, y) \in Z \times Y$, $f(z, y)>\lambda$ implies that there exists some point $z^{\prime} \in Z$ and some neighborhood $\mathcal{N}_{Y}(y)$ of $y$ in $Y$ such that $f\left(z^{\prime}, w\right)>\lambda$ for all $w \in \mathcal{N}_{Y}(y)$. A function $f: Z \times Y \mapsto \mathbb{R}$ is transfer lower semicontinuous in $y$ if $f$ is $\lambda$-transfer lower semicontinuous in $y$ for every $\lambda \in \mathbb{R}$.

A game is payoff secure if and only if each player's payoff function is transfer lower semicontinuous in the other players' strategies (Prokopovych 2011).

The graph of $G$ is defined by $\operatorname{Gr} G=\left\{(x, u) \in X \times \mathbb{R}^{n} \mid u_{i}(x)=u_{i}\right.$ for all $i \in N\}$. For a subset $B$ of a topological vector space $X$, we denote the interior of $B$ in $X$ by $\operatorname{int}_{X} B$, the boundary of $B$ by $\partial B$, the closure of $B$ by $\mathrm{cl} B$, and the convex hull of $B$ by $\operatorname{co} B$. In a metric space $Y$, we denote by $B_{Y}(y, r)$ the open ball centered at $y$ and with radius $r>0$. Denote by $E_{G}$ the set of pure strategy Nash equilibria of $G$ in $X$.

Definition 3 A game $G=\left(X_{i}, u_{i}\right)_{i \in I}$ is better-reply secure if, whenever $\left(x^{*}, u^{*}\right) \in \operatorname{clGr} G$ and $x^{*} \in X \backslash E_{G}$, some player $i$ can secure a payoff strictly above $u_{i}^{*}$ at $x^{*}$. 
Reny's (1999) equilibrium existence theorem states that every compact, quasiconcave, better-reply secure game has a Nash equilibrium in pure strategies. Better-reply security is equivalent to transfer reciprocal upper semicontinuity in payoff secure games. ${ }^{4}$

Another approach to studying the equilibrium existence problem in discontinuous games was proposed by Baye, Tian, and Zhou (1993).

For $G=\left(X_{i}, u_{i}\right)_{i \in I}$, define the following aggregator functions:

$$
A_{G}: X \times X \rightarrow \mathbb{R} \text { by } A_{G}(d, x)=\sum_{i \in I} u_{i}\left(d_{i}, x_{-i}\right),
$$

where, as usual, the $-i$ subscript on $x$ stands for "all players except $i$,"

$$
A_{G}^{0}: X \rightarrow \mathbb{R} \text { by } A_{G}^{0}(x)=\sum_{i \in I} u_{i}(x),
$$

and

$$
F_{G}: X \times X \rightarrow \mathbb{R} \text { by } F_{G}(d, x)=A_{G}(d, x)-A_{G}^{0}(x) .
$$

A strategy profile $x \in X$ is a Nash equilibrium of $G$ iff $F_{G}(d, x) \leq 0$ for all $d \in X$.

Definition 4 A game $G=\left(X_{i}, u_{i}\right)_{i \in I}$ is diagonally transfer continuous if, for every $x \in X \backslash E_{G}$, there exist some $d \in X$ and some neighborhood $\mathcal{N}_{X}(x)$ of $x$ in $X$ such that $F_{G}(d, z)>0$ for all $z \in \mathcal{N}_{X}(x)$.

It is important to notice that $G$ is diagonally transfer continuous iff $F_{G}$ is 0 -transfer lower semicontinuos in $x$.

Every payoff secure game with an upper semicontinuous $A_{G}^{0}$ is diagonally transfer continuous.

Lemma 1 If, in a game $G=\left(X_{i}, u_{i}\right)_{i \in I}$, each $u_{i}: X \rightarrow \mathbb{R}$ is transfer lower semicontinuous in $x_{-i}$ and the aggregator function $A_{G}^{0}: X \rightarrow \mathbb{R}$ is upper semicontinuous, then $G$ is diagonally transfer continuous.

Proof. We shall first show that $A_{G}(d, x)$ is transfer lower semicontinuous in $x$. Let $(d, x) \in X \times X$ and $\lambda \in \mathbb{R}$ be such that $A_{G}(d, x)>\lambda$. Then there are $\lambda_{1}, \ldots, \lambda_{n} \in \mathbb{R}$ such that $\lambda=\lambda_{1}+\ldots+\lambda_{n}$ and $u_{i}\left(d_{i}, x_{-i}\right)>\lambda_{i}$

\footnotetext{
${ }^{4}$ We refer the reader to Reny (1999), Bagh and Jofre (2006), and Prokopovych (2011) for further details regarding reciprocal upper semicontinuity and its generalizations.
} 
for all $i \in I$. Since each $u_{i}$ is transfer lower semicontinuous in $x_{-i}$, there exist $\bar{d}_{i} \in X_{i}$ and an open neighborhood $\mathcal{N}_{X_{-i}}\left(x_{-i}\right)$ of $x_{-i}$ in $X_{-i}$ such that $u_{i}\left(\bar{d}_{i}, z_{-i}\right)>\lambda_{i}$ for all $z_{-i} \in \mathcal{N}_{X_{-i}}\left(x_{-i}\right)$. Consequently, $A_{G}(\bar{d}, z)>\lambda$ for every $z \in \cap_{i \in I}\left\{X_{i} \times \mathcal{N}_{X_{-i}}\left(x_{-i}\right)\right\}$.

Since $A_{G}^{0}$ is upper semicontinuous on $X$, the transfer lower semicontinuity of $A_{G}$ in $x$ implies the transfer lower semicontinuity of $F_{G}$ in $x$. Therefore $F_{G}$ is 0 -transfer lower semicontinuous in $x$.

Now we define the mixed extension of a game $G=\left(X_{i}, u_{i}\right)_{i \in I}$. Denote by $\triangle\left(X_{i}\right)$ the set of Borel probability measures on $X_{i}$ and by $c a\left(X_{i}\right)$ the set of Borel signed measures with finite total variation on $X_{i}$. A basic open neighborhood of $\mu_{i} \in c a\left(X_{i}\right)$ in the weak topology on $c a\left(X_{i}\right)$ is a set of the form $\left\{\nu_{i} \in c a\left(X_{i}\right):\left|\int f_{j}\left(d \nu_{i}-d \mu_{i}\right)\right|<\varepsilon, j=1, \ldots, m\right\}$ for some $f_{1}, \ldots, f_{m} \in C\left(X_{i}\right)$ and $\varepsilon>0$. The set $c a\left(X_{i}\right)$ is a Hausdorff topological vector space equipped with the weak topology. The topology induced on $\triangle\left(X_{i}\right)$ by the weak topology is compact. ${ }^{5}$ Let each of the Cartesian products $c a(X)=c a\left(X_{1}\right) \times \ldots \times c a\left(X_{n}\right)$ and $\triangle(X)=\triangle\left(X_{1}\right) \times \ldots \times \triangle\left(X_{n}\right)$ be equipped with the product topology. The set $c a(X)$ is a Hausdorff topological vector space in which the operations of addition and scalar multiplication are defined as follows: for $\mu=\left(\mu_{1}, \ldots, \mu_{n}\right) \in c a(X)$ and $\alpha \in \mathbb{R}$ the scalar multiplication of $\mu$ by $\alpha$ is the element $\alpha \mu$ given by $\alpha \mu=\left(\alpha \mu_{1}, \ldots, \alpha \mu_{n}\right)$. The addition of $\mu=\left(\mu_{1}, \ldots, \mu_{n}\right) \in c a(X)$ and $v=\left(\nu_{1}, \ldots, \nu_{n}\right) \in c a(X)$ gives $\mu+v=\left(\mu_{1}+\nu_{1}, \ldots, \mu_{n}+\nu_{n}\right)$.

The mixed extension of the game $G$ is the $n$-player normal form game $\Gamma=\left(\triangle\left(X_{i}\right), U_{i}\right)_{i \in I}$, where $\triangle\left(X_{i}\right)$ is player $i$ 's strategy set and player $i$ 's payoff function $U_{i}: \triangle(X) \rightarrow \mathbb{R}$ is defined by

$$
U_{i}(\mu)=\int_{X_{1}} \int_{X_{2}} \ldots \int_{X_{n}} u_{i}\left(x_{1}, \ldots, x_{n}\right) d \mu_{1} \ldots d \mu_{n} .
$$

Since Fubini's theorem holds in this context, the integral in the definition of player $i$ 's expected payoff is properly defined.

For the game $\Gamma$, we also define the aggregator functions $A_{\Gamma}: \triangle(X) \times$ $\triangle(X) \rightarrow \mathbb{R}, A_{\Gamma}^{0}: \triangle(X) \rightarrow \mathbb{R}$, and $F_{\Gamma}: \triangle(X) \times \triangle(X) \rightarrow \mathbb{R}$ (see the corresponding definitions for $G$ ).

\footnotetext{
${ }^{5}$ In order to make $\triangle\left(X_{i}\right)$ a subset of a linear space, we embed it in the space $c a\left(X_{i}\right)$ of signed measures with finite total variation on $X_{i}$. Sometimes it is possible to proceed without the embedding (see, e.g., the proof of the compactness of the set of probability measures given by Glycopantis and Muir 2004).
} 
Theorem 1 If the mixed extension $\Gamma$ of a game $G=\left(X_{i}, u_{i}\right)_{i \in I}$ is diagonally transfer continuous, then $G$ possesses a mixed strategy Nash equilibrium.

Proof. The set $\triangle(X)$ is a compact, convex subset of $c a(X)$. Consider the aggregator function $F_{\Gamma}(\sigma, \mu): \triangle(X) \times \triangle(X) \rightarrow \mathbb{R}$. Since $F_{\Gamma}$ is linear in $\sigma$ and 0 -transfer lower semicontinuous in $\mu$, the mixed extension $\Gamma$ of $G$ has a Nash equilibrium in pure strategies by the Ky Fan minimax inequality (see Lemma 9 in the Appendix).

Another proof of Theorem 1 can be obtained by using the fact that every diagonally transfer continuous mixed extension has the single deviation property (see Reny 2009, footnote 4; Reny 2011, p. 19; and Prokopovych 2012 for some details).

Since the upper semicontinuity of $A_{G}^{0}$ implies the upper semicontinuity of $A_{\Gamma}^{0}$, verifying whether a game has a mixed strategy Nash equilibirum usually means verifying the following two properties: (a) the upper semicontinuity of the sum of the payoff functions; and (b) the payoff security of its mixed extension. If these properties hold, the mixed extension of the game is not only better-reply secure but, by Lemma 1, diagonally transfer continuous.

The diagonal transfer continuity of a game does not imply that its mixed extension is diagonally transfer continuous. For instance, Sion and Wolfe's zero-sum game (Sion and Wolfe 1957) is payoff secure (see Carmona 2005, Example 4) and its aggregator function $A_{G}^{0}$ is constant. Thus, the game is diagonally transfer continuous by Lemma 1. However, since the game has no mixed strategy Nash equilibrium, its mixed extension is not diagonally transfer continuous.

\section{Uniform Security}

An easily verifiable condition for the mixed extension of a game to be payoff secure is that of uniform payoff security, due to Monteiro and Page (2007).

Definition 5 A game $G=\left(X_{i}, u_{i}\right)_{i \in I}$ is uniformly payoff secure if, for every $x_{i} \in X_{i}$ and every $\varepsilon>0$, there is $d_{i} \in X_{i}$ such that, for every $x_{-i} \in X_{-i}$, $u_{i}\left(d_{i}, w_{-i}\right) \geq u_{i}\left(x_{i}, x_{-i}\right)-\varepsilon$ for all $w_{-i}$ in some open neighborhood $\mathcal{N}_{X_{-i}}\left(x_{-i}\right)$ of $x_{-i}$ in $X_{-i}$.

It is useful to notice that: (a) replacing the pure deviation strategy $d_{i}$ in Definition 5 with a mixed deviation strategy $\sigma_{i} \in \triangle\left(X_{i}\right)$ would not affect 
the validity of the proof of Theorem 1 of Monteiro and Page (2007); (b) uniform payoff security can be introduced in a pointwise manner. We will need the following definition. In $G=\left(X_{i}, u_{i}\right)_{i \in I}$, player $i$ 's payoff function $u_{i}: X_{i} \times X_{-i} \rightarrow \mathbb{R}$ is uniformly transfer lower semicontinuous in $x_{-i}$ at $x_{i} \in X_{i}$ if, for every $\varepsilon>0$, there is $d_{i} \in X_{i}$ such that, for every $x_{-i} \in X_{-i}$, there exists a neighborhood $\mathcal{N}_{X_{-i}}\left(x_{-i}\right)$ of $x_{-i}$ in $X_{-i}$ such that $u_{i}\left(d_{i}, w_{-i}\right)>u_{i}\left(x_{i}, x_{-i}\right)-\varepsilon$ for all $w_{-i} \in \mathcal{N}_{X_{-i}}\left(x_{-i}\right)$.

Corollary 1 If $G=\left(X_{i}, u_{i}\right)_{i \in I}$ is uniformly payoff secure and its aggregator function $A_{G}^{0}$ is upper semicontinuous, then the mixed extension $\Gamma$ is diagonally transfer continuous, and, therefore, $G$ possesses a mixed strategy Nash equilibrium.

The notion of a uniformly payoff secure game can be extended to diagonally transfer continuous games.

Definition 6 A game $G=\left(X_{i}, u_{i}\right)_{i \in I}$ is uniformly diagonally secure if, for every $d \in X$ and every $\varepsilon>0$, there is $\bar{d} \in X$ such that, for every $x \in X$, $F_{G}(\bar{d}, w)>F_{G}(d, x)-\varepsilon$ for all $w$ in some open neighborhood $\mathcal{N}_{X}(x)$ of $x$ in $X$.

An upper semicontinuous-sum game $G$ is uniformly diagonally secure if it is uniformly payoff secure.

Lemma 2 If a game $G=\left(X_{i}, u_{i}\right)_{i \in I}$ is uniformly payoff secure and the aggregator function $A_{G}^{0}: X \rightarrow \mathbb{R}$ is upper semicontinuous, then $G$ is uniformly diagonally secure.

Proof. Fix some $d \in X$. By the uniform payoff security of $G$, for every $\varepsilon>0$ and each $i \in I$, there is a deviation strategy $\bar{d}_{i} \in X_{i}$ such that, for every $x_{-i} \in X_{-i}, u_{i}\left(\bar{d}_{i}, w_{-i}\right) \geq u_{i}\left(d_{i}, x_{-i}\right)-\frac{\varepsilon}{2 n}$ for all $w_{-i}$ in some open neighborhood $\mathcal{N}_{X_{-i}}\left(x_{-i}\right)$ of $x_{-i}$ in $X_{-i}$. Consider some $x \in X$ and denote $\mathcal{N}_{X}^{1}(x)=\cap_{i \in I}\left\{X_{i} \times \mathcal{N}_{X_{-i}}\left(x_{-i}\right)\right\}$. Then $A_{G}(\bar{d}, w) \geq A_{G}(d, x)-\frac{\varepsilon}{2}$ for all $w \in$ $\mathcal{N}_{X}^{1}(x)$. Since the function $-A_{G}^{0}$ is lower semicontinuous on $X$, there exists a neighborhood $\mathcal{N}_{X}^{2}(x)$ such that $-A_{G}^{0}(w)>-A_{G}^{0}(x)-\frac{\varepsilon}{2}$ for all $w \in \mathcal{N}_{X}^{2}(x)$. Then $F_{G}(\bar{d}, w)>F_{G}(d, x)-\varepsilon$ for all $w \in \mathcal{N}_{X}^{1}(x) \cap \mathcal{N}_{X}^{2}(x)$.

Theorem 2 If a game $G=\left(X_{i}, u_{i}\right)_{i \in I}$ is uniformly diagonally secure, then its mixed extension $\Gamma$ is diagonally transfer continuous, and, therefore, $G$ possesses a mixed strategy Nash equilibrium. 
The proof of Theorem 2 follows the lines of the proof of Theorem 1 of Monteiro and Page (2007) and is given in the Appendix.

The concept of uniform diagonal security may be of help in studying equilibirum existence in games whose aggregator function $A_{G}^{0}$ is not upper semicontinuous.

Example 1 Consider a slight modification of the rent-seeking game due to Tullock (1980). Two players simultaneously bid for a political favor commonly known worth $V$ dollars. Their bids, denoted by $x_{1}$ and $x_{2}$, influence the probability of receiving the favor. Player $i$ 's strategy set is the segment $[0, V]$. Let $\pi_{i}\left(x_{1}, x_{2}\right)$ denote the probability player $i$ wins. The function $\pi_{i}$, often called player $i$ 's contest success function, is specified as follows:

$$
\pi_{i}\left(x_{1}, x_{2}\right)=\left\{\begin{array}{l}
\frac{1}{4} \text { if } x_{1}=x_{2}=0 \\
\frac{1}{2} \text { if } x_{i}>x_{-i}=0 \\
\frac{x_{i}^{r}}{x_{i}^{r}+x_{-i}^{r}} \text { otherwise }
\end{array}\right.
$$

where $r>0$. Player $i$ 's payoff function $u_{i}$ is

$$
u_{i}\left(x_{1}, x_{2}\right)=\pi_{i}\left(x_{1}, x_{2}\right) V-x_{i} .
$$

The only difference of the model from the Tullock rent-seeking game is the assumption that if the lowest bid submitted is equal to zero (or, in other words, at least one player exerts no effort at all), the favor may be granted to a third party with probability one-half. Consequently, the aggregator function $A_{G}^{0}$ is not upper semicontinuous.

Let, for specificity, $V=2$ and $r=3$. In this case, the game has no pure strategy Nash equilibria (see Baye, Kovenock, and de Vries 1994 for a related discussion). For instance, one can check that the only candidate point for being an interior solution is $(1.5,1.5)$, a strategy profile where both players get negative expected payoffs. However, each of them can avoid getting a negative payoff by bidding zero.

To verify that the game is not better-reply secure, consider the sequence $\left\{x^{k}\right\}$ with $x^{k}=\left(\frac{1}{k}, \frac{1}{k}\right)$ for $k=1,2, \ldots$ Then the corresponding sequence of payoff vectors $\left\{\left(u_{1}\left(x^{k}\right), u_{2}\left(x^{k}\right)\right)\right\}$ converges to $(1,1)$. It is clear that no player can secure a payoff strictly above 1 at $(0,0)$.

On the other hand, the game is not only diagonally transfer continuous, it is uniformly diagonally secure (see the Appendix for details). Therefore, the game possesses a mixed strategy Nash equilibrium. 


\section{Uniform Hospitality}

In this section we extend the notion of a hospitable strategy, initially developed by Duggan (2007) for zero-sum games, to our framework.

Definition 7 A strategy $\sigma_{i} \in \triangle\left(X_{i}\right)$ of player $i$ is called hospitable if $U_{i}\left(\sigma_{i}, \cdot\right)$ : $X_{-i} \rightarrow \mathbb{R}$ defined by $U_{i}\left(\sigma_{i}, x_{-i}\right)=\int_{X_{i}} u_{i}\left(x_{i}, x_{-i}\right) d \sigma_{i}$ is continuous.

Definition 8 A game $G=\left(X_{i}, u_{i}\right)_{i \in I}$ is called hospitable if, for each $i \in I$, every $\mu \in \triangle(X)$, and every $\varepsilon>0$, there is a hospitable strategy $\sigma_{i}$ such that $U_{i}\left(\sigma_{i}, \mu_{-i}\right)>U_{i}(\mu)-\varepsilon$.

There is no ambiguity associated with the notation used in Definition 7 since $U_{i}\left(\sigma_{i}, \mu_{-i}\right)=\int_{X_{-i}} U_{i}\left(\sigma_{i}, x_{-i}\right) d \mu_{-i}$ by Fubini's theorem.

Lemma 3 If $G=\left(X_{i}, u_{i}\right)_{i \in I}$ is a hospitable game, then its mixed extension $\Gamma$ is payoff secure.

Proof. Fix some $\mu=\left(\mu_{1}, \ldots, \mu_{n}\right) \in \triangle(X), \varepsilon>0$, and $i \in I$. We have to show that there are a strategy $\sigma_{i} \in \triangle\left(X_{i}\right)$ and a neighborhood $\mathcal{N}_{\triangle\left(X_{-i}\right)}\left(\mu_{-i}\right)$ such that $U_{i}\left(\sigma_{i}, \mu_{-i}^{\prime}\right) \geq U_{i}(\mu)-\varepsilon$ for all $\mu_{-i}^{\prime} \in \mathcal{N}_{\triangle\left(X_{-i}\right)}\left(\mu_{-i}\right)$.

Since $G$ is a hospitable game, $U_{i}\left(\sigma_{i}, \mu_{-i}\right)>U_{i}(\mu)-\varepsilon$ for some hospitable $\sigma_{i} \in \triangle\left(X_{i}\right)$. Since $U_{i}\left(\sigma_{i}, \cdot\right): X_{-i} \rightarrow \mathbb{R}$ is continuous, $U_{i}\left(\sigma_{i}, \cdot\right): \triangle\left(X_{-i}\right) \rightarrow$ $\mathbb{R}$ is continuous as well (see Aliprantis and Border 2006, Theorem 15.5). Therefore, there exists a neighborhood $\mathcal{N}_{\triangle\left(X_{-i}\right)}\left(\mu_{-i}\right)$ such that $U_{i}\left(\sigma_{i}, \mu_{-i}^{\prime}\right)>$ $U_{i}(\mu)-\varepsilon$ for all $\mu_{-i}^{\prime} \in \mathcal{N}_{\triangle\left(X_{-i}\right)}\left(\mu_{-i}\right)$.

Corollary 2 If $G=\left(X_{i}, u_{i}\right)_{i \in I}$ is a hospitable game with an upper semicontinuous aggregator function $A_{G}^{0}$, then it has a mixed strategy Nash equilibrium.

We now give some sufficient conditions for a game to be hospitable.

Definition 9 A game $G=\left(X_{i}, u_{i}\right)_{i \in I}$ is uniformly hospitable if, for each $i \in$ $I$, every $x_{i} \in X_{i}$, and every $\varepsilon>0$, there is a hospitable strategy $\sigma_{i} \in \triangle\left(X_{i}\right)$ such that $U_{i}\left(\sigma_{i}, x_{-i}\right)>u_{i}\left(x_{i}, x_{-i}\right)-\varepsilon$ for every $x_{-i} \in X_{-i}$.

Lemma 4 Every uniformly hospitable game $G=\left(X_{i}, u_{i}\right)_{i \in I}$ is hospitable. 
Proof. Fix some $i \in\{1, \ldots, n\}, \mu \in \triangle(X)$, and $\varepsilon>0$. We have to show that there is a hospitable strategy $\sigma_{i}$ such that $U_{i}\left(\sigma_{i}, \mu_{-i}\right)>U_{i}(\mu)-\varepsilon$.

First, pick a strategy $x_{i} \in X_{i}$ such that $U_{i}\left(x_{i}, \mu_{-i}\right) \geq U_{i}(\mu)$. Since $G$ is uniformly hospitable, there exists a hospitable strategy $\sigma_{i} \in \triangle\left(X_{i}\right)$ such that $U_{i}\left(\sigma_{i}, \mu_{-i}\right)>U_{i}\left(x_{i}, \mu_{-i}\right)-\varepsilon$. Therefore $U_{i}\left(\sigma_{i}, \mu_{-i}\right)>U_{i}(\mu)-\varepsilon$.

From now on, we impose the additional assumption that each $X_{i}$ is a subset of a finite-dimensional Euclidean space. Denote by $\lambda_{i}$ the Lebesgue measure on $X_{i}$. The proof of the next lemma is based on some ideas from Duggan (2007, Propositions 2 and 3) and is relegated to the Appendix.

Lemma 5 Let each $X_{i}, i \in I$, be a compact subset of a finite-dimensional Euclidean space. A game $G=\left(X_{i}, u_{i}\right)_{i \in I}$ is uniformly hospitable if

(i) for each $i \in N$ and every $x_{-i} \in X_{-i}$,

$$
\lambda_{i}\left(\left\{x_{i} \in X_{i}: u_{i}\left(x_{i}, \cdot\right) \text { is discontinuous at } x_{-i}\right\}\right)=0,
$$

(ii) for each $i \in N$, every $x_{i} \in X_{i}$, and every $\varepsilon>0$, there is an open ball $B_{i}^{+}\left(x_{i}, \varepsilon\right)$ in $X_{i}$ such that $u_{i}\left(x_{i}^{\prime}, x_{-i}\right)>u_{i}\left(x_{i}, x_{-i}\right)-\varepsilon$ for every $x_{i}^{\prime} \in B_{i}^{+}\left(x_{i}, \varepsilon\right)$ and every $x_{-i} \in X_{-i}$.

It is worth noticing that $B_{i}^{+}\left(x_{i}, \varepsilon\right)$ is not necessarily centered at $x_{i}$.

Assumption (i) of Lemma 5 is akin to the one-to-one assumption used by Dasgupta and Maskin (1986, definitions on p. 7 and Example 4 on p. 21) and is not superfluous.

Example 2 Consider $G=\left(X_{i}, u_{i}\right)_{i \in\{1,2\}}$ where $X_{1}=X_{2}=[0,1]$ and player $i$ 's payoff function is defined as follows:

$$
\begin{aligned}
& u_{1}\left(x_{1}, x_{2}\right)=\left\{\begin{array}{l}
x_{1} \text { if }\left(x_{1}, x_{2}\right) \in[0,1] \times[0,1) \\
2-x_{1} \text { if }\left(x_{1}, x_{2}\right) \in[0,1] \times\{1\}
\end{array}\right. \\
& u_{2}\left(x_{1}, x_{2}\right)=\left\{\begin{array}{l}
x_{2} \text { if }\left(x_{1}, x_{2}\right) \in(0,1] \times[0,1] \text { and }\left(x_{1}, x_{2}\right)=(0,1), \\
3-x_{2} \text { if }\left(x_{1}, x_{2}\right) \in\{0\} \times[0,1) .
\end{array}\right.
\end{aligned}
$$

It is easy to see that the sum of the payoff functions is upper semicontinuous on $X$. The game is compact, quasiconcave, and satisfies assumption (ii) of Lemma 5. However it has no mixed strategy Nash equilibria. To show this, assume, by way of contradiction, that the game has a mixed strategy Nash equilibrium, $\bar{\mu}=\left(\bar{\mu}_{1}, \bar{\mu}_{2}\right)$. For each $i \in\{1,2\}$ define $B R_{i}: \triangle\left(X_{-i}\right) \rightarrow \triangle\left(X_{i}\right)$ by

$$
B R_{i}\left(\mu_{-i}\right)=\left\{\sigma_{i} \in \triangle\left(X_{i}\right): U_{i}\left(\sigma_{i}, \mu_{-i}\right) \geq U_{i}\left(\mu_{i}, \mu_{-i}\right) \text { for every } \mu_{i} \in \triangle\left(X_{i}\right)\right\} .
$$


Since $\bar{\mu}$ is a Nash equilibrium of $\Gamma, \bar{\mu}_{i} \in B R_{i}\left(\bar{\mu}_{-i}\right)$ for $i=1,2$. Denote by $\delta_{x}$ the Dirac measure concentrated at $x \in[0,1]$.

If $\bar{\mu}_{1}(\{0\})=0$, then $B R_{2}\left(\bar{\mu}_{1}\right)=\delta_{1}$. However, if $\bar{\mu}_{2}=\delta_{1}$, then $B R_{1}\left(\bar{\mu}_{2}\right)=$ $\delta_{0}$, and, therefore, $\bar{\mu}_{1}(\{0\})=1$, a contradiction.

If $\bar{\mu}_{2}(\{1\})=0$, then $B R_{1}\left(\bar{\mu}_{2}\right)=\delta_{1}$, which, in turn, implies that $B R_{2}\left(\bar{\mu}_{1}\right)=$ $\delta_{1}$, another impossibility.

Therefore, both $\bar{\mu}_{1}(\{0\})>0$ and $\bar{\mu}_{2}(\{1\})>0$. Then the fact that $\bar{\mu}_{2} \in B R_{2}\left(\bar{\mu}_{1}\right)$ implies that $U_{2}\left(\bar{\mu}_{1}, \delta_{1}\right) \geq U_{2}\left(\bar{\mu}_{1}, \mu_{2}\right)$ for every $\mu_{2} \in \triangle\left(X_{2}\right)$. By definition, $U_{2}\left(\bar{\mu}_{1}, \delta_{1}\right)=\bar{\mu}_{1}(\{0\}) u_{2}(0,1)+\int_{(0,1]} u_{2}\left(x_{1}, 1\right) d \mu_{1}$. Fix some $\varepsilon \in\left(0, \bar{\mu}_{1}(\{0\})\right)$ and pick some $x_{2}^{\prime}$ close enough to one such that $u_{2}\left(x_{1}, x_{2}^{\prime}\right)>$ $u_{2}\left(x_{1}, 1\right)-\varepsilon$ for every $x_{1} \in[0,1]$. Then $U_{2}\left(\bar{\mu}_{1}, \delta_{x_{2}^{\prime}}\right)-U_{2}\left(\bar{\mu}_{1}, \delta_{1}\right)>\bar{\mu}_{1}(\{0\})\left(u_{2}\left(0, x_{1}^{\prime}\right)-\right.$ $\left.u_{2}(0,1)\right)-\varepsilon>0$, which contradicts the initial premise that $\bar{\mu}$ is a Nash equilibrium of $\Gamma$.

In finite-dimensional applications, it is sometimes possible to apply the notion of a uniformly hospitable game in place of the notion of a uniformly payoff secure game.

Example 3 Consider the following uniformly payoff secure game $G=$ $\left(X_{i}, u_{i}\right)_{i \in I}$ (see Monteiro and Page 2007, Example 1; Baye, Kovenock, and de Vries 1996).

There are $n$ bidders, competing for an object with a known value of unity. Each player $i$ submits a sealed bid $b_{i} \in[0,1]$. Let $b^{*}=\max _{i \in I} b_{i}$, $H=\left\{i \in I: b_{i}=b^{*}\right\}$, and $|H|$ be the cardinality of $H$. Bidder $i$ 's payoff is as follows:

$$
u_{i}(b)=\left\{\begin{array}{l}
\frac{1}{|H|}-b_{i} \text { if } b_{i}=b^{*} \\
-b_{i} \text { if } b_{i}<b^{*}
\end{array}\right.
$$

Let $\varepsilon \in(0,1)$ and $b_{i} \in X_{i}=[0,1]$. To show that the auction is uniformly hospitable, we have to consider two cases.

Case 1. If $b_{i} \in[0,1)$, pick $\bar{b}_{i}>b_{i}$ and $\eta>0$ such that $b_{i}<\bar{b}_{i}-\eta$ and $\bar{b}_{i}+\eta<\min \left\{1, b_{i}+\varepsilon\right\}$. Put $B_{i}^{+}\left(b_{i}, \varepsilon\right)=B_{X_{i}}\left(\bar{b}_{i}, \eta\right)$.

Case 2. If $b_{i}=1$, then player $i$ 's payoff is either zero or negative. Put $B_{i}^{+}\left(x_{i}, \varepsilon\right)=B_{X_{i}}(0, \varepsilon)$.

It is clear that in each of the cases $u_{i}\left(\bar{b}_{i}, b_{-i}\right)>u_{i}\left(b_{i}, b_{-i}\right)-\varepsilon$ for every $\bar{b}_{i} \in S_{i}^{+}\left(b_{i}, \varepsilon\right)$ and every $b_{-i} \in X_{-i}$. By Lemma 5 , the game is uniformly hospitable. 


\section{Uniform Conditions in Two Player Games on the Unit Square}

Theorem 5b of Dasgupta and Maskin (1986) (hereinafter called Theorem $5 \mathrm{~b})$ is an important equilibrium existence result in which the assumption that the sum of the players' payoffs functions is upper semicontinuous is relaxed. As shown by Bagh (2010) with the aid of examples, the proof of Theorem 5b is not in full harmony with its statement. The modification of the theorem proposed by Bagh (2010, Theorem 5.1) is applicable mostly to Bertrand-Edgeworth games (see also Remark 1 below). Making use of the concepts of uniform payoff security and uniform hospitality leads us to another modification of Theorem 5b.

We consider a two-player game $G=\left(X_{i}, u_{i}\right)_{i \in\{1,2\}}$ on the unit square, $X=$ $X_{1} \times X_{2}=[0,1] \times[0,1]$, where the payoff functions' discontinuities lie on the main diagonal of $X$. For such games, we will need the following strengthening of uniform hospitality. Player $i$ 's payoff function $u_{i}:[0,1] \times[0,1] \rightarrow \mathbb{R}$ is called strongly uniformly hospitable at $x_{i} \in[0,1]$ if, for every $\varepsilon>0$, there exists $\delta(\varepsilon) \in \mathbb{R} \backslash\{0\}$ such that $x_{i}+\delta(\varepsilon) \in[0,1]$ and, for every $d_{i} \in\left(x_{i}, x_{i}+\delta(\varepsilon)\right)$, $u_{i}\left(d_{i}, x_{-i}\right)>u_{i}\left(x_{i}, x_{-i}\right)-\varepsilon$ for all $x_{-i} \in X_{-i}$. The game $G=\left(X_{i}, u_{i}\right)_{i \in\{1,2\}}$ is strongly uniformly hospitable if each $u_{i}$ is strongly uniformly hospitable at every $x_{i} \in X_{i}$.

The set $X$ consists of three subsets: $S^{1}=\left\{\left(x_{1}, x_{2}\right) \in[0,1] \times[0,1]: x_{1} \in\right.$ $[0,1]$ and $\left.x_{2}>x_{1}\right\}, S^{2}=\left\{\left(x_{1}, x_{2}\right) \in[0,1] \times[0,1]: x_{1} \in[0,1]\right.$ and $\left.x_{1}>x_{2}\right\}$, and $S=\left\{\left(x_{1}, x_{2}\right):[0,1] \times[0,1]: x_{1}=x_{2}\right\}$.

Lemma 6 Consider a two-player game $G=\left(X_{i}, u_{i}\right)_{i \in\{1,2\}}$ on the unit square $X=[0,1] \times[0,1]$. Suppose that there are continuous functions $l_{i}^{j}: \operatorname{cl}^{j} \rightarrow \mathbb{R}$, $i=1,2, j=1,2$ such that $u_{i}(x)=l_{i}^{j}(x)$ for all $x \in S^{j}$ and all $i, j \in\{1,2\}$. If player $i$ 's payoff function $u_{i}$ is strongly uniformly hospitable at some $x_{i} \in$ $[0,1]$, then it is uniformly transfer lower semicontinuous in $x_{-i}$ at $x_{i}$.

Since each $l_{i}^{j}$ is defined on $\operatorname{cl} S^{j}, \lim _{k \rightarrow \infty} u_{i}\left(x^{k}\right)=l_{i}^{j}(x)$ for every sequence $\left\{x^{k}\right\} \subset S^{j}$ converging to $x \in S$.

Proof. Since $u_{i}$ is strongly uniformly hospitable at $x_{i}$, for every $\varepsilon>0$ there exists $\delta(\varepsilon) \in \mathbb{R} \backslash\{0\}$ such that $x_{i}+\delta(\varepsilon) \in[0,1]$ and, for every $d_{i} \in$ $\left(x_{i}, x_{i}+\delta(\varepsilon)\right), u_{i}\left(d_{i}, x_{-i}\right)>u_{i}\left(x_{i}, x_{-i}\right)-\varepsilon$ for all $x_{-i} \in X_{-i}$. Fix some arbitrary $\varepsilon>0$ and some $d_{i} \in\left(x_{i}, x_{i}+\delta\left(\frac{\varepsilon}{2}\right)\right)$. We have to show that, for 
every $x_{-i} \in X_{-i}$, there exists a neighborhood $\mathcal{N}_{X_{-i}}\left(x_{-i}\right)$ of $x_{-i}$ in $X_{-i}$ such that $u_{i}\left(d_{i}, w_{-i}\right)>u_{i}\left(x_{i}, x_{-i}\right)-\varepsilon$ for all $w_{-i} \in \mathcal{N}_{X_{-i}}\left(x_{-i}\right)$.

If $x_{-i} \neq d_{i}$, then $u_{i}$ is continuous at $\left(d_{i}, x_{-i}\right)$, and we can choose a suitable neighborhood $\mathcal{N}_{X_{-i}}\left(x_{-i}\right)$. If $x_{-i}=d_{i}$, then $\left(x_{i}, x_{-i}\right) \in S^{j}$ for some $j \in\{1,2\}$. Pick $r>0$ such that $\left(x_{i}, w_{-i}\right) \in S^{j}$ and $\left|u_{i}\left(x_{i}, w_{-i}\right)-u_{i}\left(x_{i}, x_{-i}\right)\right|<\frac{\varepsilon}{2}$ for all $w_{-i} \in B_{X_{-i}}\left(x_{-i}, r\right)$. Then $u_{i}\left(d_{i}, w_{-i}\right)>u_{i}\left(x_{i}, w_{-i}\right)-\frac{\varepsilon}{2}>u_{i}\left(x_{i}, x_{-i}\right)-\varepsilon$ for every $w_{-i} \in B_{X_{-i}}\left(x_{-i}, r\right)$.

The next lemma states that any game covered by Theorem $5 \mathrm{~b}$ is uniformly payoff secure.

Lemma 7 Consider a two-player game $G=\left(X_{i}, u_{i}\right)_{i \in\{1,2\}}$ on the unit square $X=[0,1] \times[0,1]$. Assume that

(i) there are continuous functions $l_{i}^{j}: \operatorname{cl}^{j} \rightarrow \mathbb{R}, i=1,2, j=1,2$ such that $u_{i}(x)=l_{i}^{j}(x)$ for all $x \in S^{j}$ and all $i, j \in\{1,2\}$;

(ii) for each $i \in\{1,2\}$ and every $x \in S$, there exists $j \in\{1,2\}$ such that

$$
l_{i}^{j}(x) \geq u_{i}(x) \geq l_{i}^{-j}(x)
$$

Then $G$ is uniformly payoff secure.

Proof. Fix some $i \in\{1,2\}, x_{i} \in(0,1)$, and $\varepsilon>0$. Then, by (ii), there exists $j \in\{1,2\}$ such that $l_{i}^{j}\left(x_{i}, x_{i}\right) \geq u_{i}\left(x_{i}, x_{i}\right) \geq l_{i}^{-j}\left(x_{i}, x_{i}\right)$. Suppose that $i=1$ and $j=2$. The rest of the cases can be handled similarly.

Since $l_{1}^{1}$ and $l_{1}^{2}$ are uniformly continuous on their respective compact domains, there exists $\delta \in\left(0, \min \left\{x_{1}, 1-x_{1}\right\}\right)$ such that, for each $j \in\{1,2\}$, $\left|l_{1}^{j}\left(x^{\prime}\right)-l_{1}^{j}\left(x^{\prime \prime}\right)\right|<\frac{\varepsilon}{2}$ for all $x^{\prime}$ and $x^{\prime \prime}$ in $\operatorname{cl} S^{j}$ with $\left\|x^{\prime}-x^{\prime \prime}\right\|<\delta$, where $\left\|x^{\prime}-x^{\prime \prime}\right\|$ denotes the Euclidean distance between $x^{\prime}$ and $x^{\prime \prime}$.

We shall show that, for any fixed $d_{1} \in\left(x_{1}, x_{1}+\delta\right), u_{1}\left(d_{1}, x_{2}\right)>u_{1}\left(x_{1}, x_{2}\right)-$ $\varepsilon$ for all $x_{2} \in X_{2}$. If $x_{2} \in X_{2} \backslash\left[x_{1}, d_{1}\right]$, then $\left(d_{1}, x_{2}\right)$ and $\left(x_{1}, x_{2}\right)$ lie in the same $S^{j}$ and, therefore, $u_{1}\left(d_{1}, x_{2}\right)>u_{1}\left(x_{1}, x_{2}\right)-\varepsilon$. If $x_{2}=x_{1}$, then $u_{1}\left(x_{1}, x_{1}\right) \leq l_{1}^{2}\left(x_{1}, x_{1}\right)<l_{1}^{2}\left(d_{1}, x_{1}\right)+\varepsilon=u_{1}\left(d_{1}, x_{1}\right)+\varepsilon$. If $x_{2} \in\left(x_{1}, d_{1}\right]$, then $u_{1}\left(x_{1}, x_{2}\right)<l_{1}^{1}\left(x_{1}, x_{1}\right)+\frac{\varepsilon}{2} \leq l_{1}^{2}\left(x_{1}, x_{1}\right)+\frac{\varepsilon}{2}<l_{1}^{2}\left(d_{1}, x_{2}\right)+\varepsilon$. Therefore, $u_{1}\left(x_{1}, x_{2}\right)<u_{1}\left(d_{1}, x_{2}\right)+\varepsilon$ for all $x_{2} \in\left(x_{1}, d_{1}\right)$. If $x_{2}=d_{1}$, then $u_{1}\left(x_{1}, d_{1}\right)=l_{1}^{1}\left(x_{1}, d_{1}\right)<l_{1}^{1}\left(d_{1}, d_{1}\right)+\frac{\varepsilon}{2}$. Then $u_{1}\left(x_{1}, d_{1}\right)<\min \left\{l_{1}^{1}\left(d_{1}, d_{1}\right)+\right.$ $\left.\varepsilon, l_{1}^{2}\left(d_{1}, d_{1}\right)+\varepsilon\right\} \leq u_{1}\left(d_{1}, d_{1}\right)+\varepsilon$.

Thus we conclude that each $u_{i}$ is strongly uniformly hospitable at every $x_{i} \in(0,1)$, which, by Lemma 6 , implies that $u_{i}$ is uniformly transfer lower semicontinuous in $x_{-i}$ at every $x_{i} \in(0,1)$. 
It is not difficult to see that $u_{i}$ need not be strongly uniformly hospitable at $x_{i}=0$ or at $x_{i}=1$. However it is still uniformly transfer lower semicontinuous in $x_{-i}$ at those $x_{i}$ 's. For instance, if $x_{i}=0$ and $l_{i}^{i}(0,0) \geq u_{i}(0,0) \geq l_{i}^{-i}(0,0)$, then put $d_{i}=0$. It is clear that for every $\varepsilon>0$ and every $x_{-i} \in X_{-i}$, there exists a neighborhood $\mathcal{N}_{X_{-i}}\left(x_{-i}\right)$ of $x_{-i}$ in $X_{-i}$ such that $u_{i}\left(0, w_{-i}\right) \geq u_{i}\left(0, x_{-i}\right)-\varepsilon$ for all $w_{-i} \in \mathcal{N}_{X_{-i}}\left(x_{-i}\right)$. If $l_{i}^{i}(0,0) \leq u_{i}(0,0) \leq l_{i}^{-i}(0,0)$, then it is not difficult to show that $u_{i}$ is strongly uniformly hospitable at $x_{i}=0$ by repeating the above argument.

In addition to all-pay auctions, Lemma 7 is applicable to first-price and second-price sealed-bid auctions, a number of location games and voting models. If the hypotheses of Lemma 7 are satisfied for a game and the game is upper semicontinuous-sum, then it has a mixed strategy Nash equilibrium.

Another important corollary of Lemma 7 is that modifications of payoffs similar to those used by Dasgupta and Maskin (1986) in the proof of Theorem $5 \mathrm{~b}$ do not lead out of the class of uniformly payoff secure games.

We need one more auxiliary lemma. Denote $S_{D}=\left\{x \in X: \limsup _{y \rightarrow x} A_{G}^{0}(y)>\right.$ $\left.A_{G}^{0}(x)\right\}$.

Lemma 8 Consider a two-player game $G=\left(X_{i}, u_{i}\right)_{i \in\{1,2\}}$ on the unit square $X=[0,1] \times[0,1]$. Suppose that the hypotheses of Lemma 7 hold and

(i) for every point $x=(z, z) \in S_{D}$, there exist $i, j \in\{1,2\}$ and some sequence $\left\{\left(x_{i}^{k}, z\right)\right\} \subset S^{j}$ converging to $x$ such that $\lim _{n \rightarrow \infty} l_{i}^{j}\left(x_{i}^{k}, z\right)>u_{i}(x)$;

(ii) if, for some $i, j \in\{1,2\}$ and some sequence $\left\{\left(x_{i}^{k}, z\right)\right\} \subset S^{j}$ converging to $x=(z, z) \in S_{D}, \lim _{n \rightarrow \infty} l_{i}^{j}\left(x_{i}^{k}, z\right)>u_{i}(x)$, then $\lim _{n \rightarrow \infty} l_{-i}^{j}\left(z, x_{i}^{k}\right)<u_{-i}(x)$.

Then each $u_{i}$ is strongly uniformly hospitable at any $z \in X_{i}$ such that $(z, z) \in$ $S_{D}$

Proof. As we have shown in the proof of Lemma 7, each $u_{i}$ is strongly uniformly hospitable at every $x_{i} \in(0,1)$. Let $(0,0) \in S_{D}$ (the argument is similar if $\left.(1,1) \in S_{D}\right)$. We shall show that both $u_{1}$ and $u_{2}$ are strongly uniformly hospitable at 0 . Since $(0,0) \in S_{D}, l_{i}^{-i}(0,0)>u_{i}(0,0)$ for some $i \in\{1,2\}$ by (i). Then $l_{-i}^{-i}(0,0)<u_{-i}(0,0)$ by (ii). Therefore, $l_{i}^{-i}(0,0) \geq$ $u_{i}(0,0) \geq l_{i}^{i}(0,0)$ and $l_{-i}^{i}(0,0) \geq u_{-i}(0,0) \geq l_{-i}^{-i}(0,0)$, which implies that each $u_{i}$ is strongly uniformly hospitable at 0 .

The following result is a modification of Theorem $5 \mathrm{~b}$.

Theorem 3 Consider a two-player game $G=\left(X_{i}, u_{i}\right)_{i \in\{1,2\}}$ on the unit square $X=[0,1] \times[0,1]$ in which the restriction of $A_{G}^{0}$ to $S$ is a continuous function from $S$ to $\mathbb{R}$. Assume that 
(i) there are continuous functions $l_{i}^{j}: \operatorname{cl}^{j} \rightarrow \mathbb{R}, i=1,2, j=1,2$ such that $u_{i}(x)=l_{i}^{j}(x)$ for all $x \in S^{j}$ and all $i, j \in\{1,2\}$;

(ii) for each $i \in\{1,2\}$ and every $x \in S$, there exists $j \in\{1,2\}$ such that

$$
l_{i}^{j}(x) \geq u_{i}(x) \geq l_{i}^{-j}(x)
$$

(iii) for every point $x=(z, z) \in S_{D}$, there exist $i, j \in\{1,2\}$ and some sequence $\left\{\left(x_{i}^{k}, z\right)\right\} \subset S^{j}$ converging to $x$ such that $\lim _{n \rightarrow \infty} l_{i}^{j}\left(x_{i}^{k}, z\right)>u_{i}(x)$; (iv) if, for some $i, j \in\{1,2\}$ and some sequence $\left\{\left(x_{i}^{k}, z\right)\right\} \subset S^{j}$ converging to $x=(z, z) \in S_{D}, \lim _{n \rightarrow \infty} l_{i}^{j}\left(x_{i}^{k}, z\right)>u_{i}(x)$, then $\lim _{n \rightarrow \infty} l_{-i}^{j}\left(z, x_{i}^{k}\right)<u_{-i}(x)$. Then $G$ has a mixed strategy Nash equilibrium.

As shown by Example A.1 of Bagh (2010), Theorem 5b lacks an assumption regarding the players' payoffs along the main diagonal of the unit square. The extra assumption that the restriction of $A_{G}^{0}$ to $S$ is a continuous function plays a crucial role in Theorem 3 . At the same time, some hypotheses of Theorem 3 are weaker than their counterparts in Theorem 5b. For instance, (iii) and (iv) are to be held only on $S_{D}$, not at every point of discontinuity.

Proof. It is not difficult to see that $S_{D}=\left\{x \in S: \max _{j \in\{1,2\}}\left\{l_{1}^{j}(x)+l_{2}^{j}(x)\right\}>\right.$ $\left.A_{G}^{0}(x)\right\}$, and, therefore, it is a relatively open subset of $S$. In particular, the latter implies that $S_{D}$ is a Borel subset of $X$. Another useful fact is the following: if $l_{i}^{j}(x)>u_{i}(x)$ for some $x=(z, z) \in S_{D}$ and some $i, j \in\{1,2\}$, then $\lim _{n \rightarrow \infty} l_{i}^{j}\left(x_{i}^{n}, z\right)>u_{i}(x)$ for some sequence $\left\{\left(x_{i}^{n}, z\right)\right\} \subset S^{j}$ converging to $x$, and, therefore, $u_{-i}(x)>l_{-i}^{j}(x)$ by (iv) (see the proof of Lemma 8 for an explanation).

The plan of the proof is similar to that used by Dasgupta and Maskin (1986, Theorem 5b). First we will modify payoffs on $S_{D}$ so as to make the sum of the payoff functions upper semicontinuous on $X$.

Define the modified payoff functions as follows: for $i \in\{1,2\}, \widehat{u}_{i}(x)=$ $u_{i}(x)$ for all $x \in X \backslash S_{D}$, and

$$
\widehat{u}_{i}(x)=\max \left\{u_{i}(x), \max _{j \in\{1,2\}}\left\{l_{i}^{j}(x)-\max \left\{0, u_{-i}(x)-l_{-i}^{j}(x)\right\}\right\}\right\} \text { for } x \in S_{D} .
$$

Since $S_{D}$ is Borel, each $\widehat{u}_{i}$ is Borel measurable on $X$. By Lemma 7, the game $\widehat{G}=\left(X_{i}, \widehat{u}_{i}\right)_{i \in\{1,2\}}$ is uniformly payoff secure.

In order to show that $A_{\widehat{G}}^{0}(x)$ is upper semicontinuous at every $x \in \operatorname{cl} S_{D}$, consider a sequence $\left\{x^{k}\right\} \subset X$ converging to $x \in \operatorname{cl} S_{D}$ such that $\lim _{k \rightarrow \infty} A_{\widehat{G}}^{0}\left(x^{k}\right)$ 
exists. We have to show that $\lim _{k \rightarrow \infty} A_{\widehat{G}}^{0}\left(x^{k}\right) \leq A_{\widehat{G}}^{0}(x)$ for any such sequence. We have to consider two cases: (a) $x \in S_{D}$; and (b) $x \in \operatorname{rbd} S_{D}$, where $\operatorname{rbd} S_{D}$ denotes the boundary of $S_{D}$ in $S$. Define $J_{i}: S \rightarrow\{1,2\}$ by $J_{i}(x)=\left\{j \in\{1,2\}: l_{i}^{j}(x)>u_{i}(x)\right.$ and $\left.l_{i}^{j}(x)-u_{i}(x)>u_{-i}(x)-l_{-i}^{j}(x)\right\}$. Notice that values of $J_{i}$ might be empty.

Case 1. Let $x \in S_{D}$. Consider a sequence $\left\{x^{k}\right\}$ lying in some $S^{j}$. Since $A_{\widehat{G}}^{0}\left(x^{k}\right)=A_{G}^{0}\left(x^{k}\right)$ for each $k$, we have that $\lim _{k \rightarrow \infty} A_{\widehat{G}}^{0}\left(x^{k}\right)=l_{1}^{j}(x)+l_{2}^{j}(x)$. If $l_{1}^{j}(x)+l_{2}^{j}(x) \leq u_{1}(x)+u_{2}(x)$, then $\lim _{k \rightarrow \infty} A_{\widehat{G}}^{0}\left(x^{k}\right) \leq A_{G}^{0}(x) \leq A_{\widehat{G}}^{0}(x)$. If $l_{1}^{j}(x)+l_{2}^{j}(x)>u_{1}(x)+u_{2}(x)$, then $j \in J_{i}(x)$ for some $i \in\{1,2\}$. By (iv), $u_{-i}(x)-l_{-i}^{j}(x)>0$, and, therefore, $\widehat{u}_{i}(x)=l_{i}^{j}(x)+l_{-i}^{j}(x)-u_{-i}(x)$. Then $\widehat{u}_{i}(x)+\widehat{u}_{-i}(x) \geq \widehat{u}_{i}(x)+u_{-i}(x)=l_{1}^{j}(x)+l_{2}^{j}(x)$.

Now consider a sequence $\left\{x^{k}\right\}$ lying in $S_{D}$. Since $x \in S_{D}, J_{i}(x) \neq \varnothing$ for at least one $i \in\{1,2\}$. Let us show that each $J_{i}$ has open lower sections; that is, if $j \in J_{i}(x)$, then $j \in J_{i}(w)$ for all $w$ in some open neighborhood $\mathcal{N}_{S}(x)$ of $x$ in $S$. Assume, by way of contradiction, that, despite the fact that $\left\{x^{k}\right\}$ tends to $x$ and $j \in J_{i}(x), j \notin J_{i}\left(x^{k}\right)$ for each $k$. Since the restriction of $A_{G}^{0}$ to $S$ is continuous, there is no loss in generality to assume that $l_{i}^{j}\left(x^{k}\right)-u_{i}\left(x^{k}\right)>$ $u_{-i}\left(x^{k}\right)-l_{-i}^{j}\left(x^{k}\right)$ for each $k$. On the other hand, since $l_{i}^{j}$ and $l_{i}^{-j}$ are continuous on $S$ and $l_{i}^{j}(x)>u_{i}(x) \geq l_{i}^{-j}(x)$, there is no loss of generality to assume that $l_{i}^{j}\left(x^{k}\right) \geq u_{i}\left(x^{k}\right) \geq l_{i}^{-j}\left(x^{k}\right)$ for all $k$. Then $j \notin J_{i}\left(x^{k}\right)$ for each $k$ only if $l_{i}^{j}\left(x^{k}\right)=u_{i}\left(x^{k}\right)$ for each $k$. Therefore, $0>u_{-i}\left(x^{k}\right)-l_{-i}^{j}\left(x^{k}\right)$ for each $k$. However, by (iv), $l_{i}^{j}\left(x^{k}\right)<u_{i}\left(x^{k}\right)$ for each $k$, a contradiction. Thus, we assume that if $j \in J_{i}(x)$ for some $i, j \in\{1,2\}$, then $j \in J_{i}\left(x^{k}\right)$ for all $k$.

We have to consider two cases: (a) $J_{1}(x) \neq \varnothing$ and $J_{2}(x) \neq \varnothing$; and (b) $J_{i}(x) \neq\{\varnothing\}$ for some $i$ and $J_{-i}(x)=\{\varnothing\}$.

If $J_{1}(x) \neq\{\varnothing\}$ and $J_{2}(x) \neq\{\varnothing\}$, then $\widehat{u}_{1}(x)+\widehat{u}_{2}(x)=\sum_{t \in\{1,2\}}\left(l_{1}^{t}(x)+\right.$ $\left.l_{2}^{t}(x)\right)-u_{1}(x)-u_{2}(x)$. Since the restriction of $A_{G}^{0}$ to $S$ is continuous and each $J_{i}$ has open lower sections, $\lim _{k \rightarrow \infty} A_{\widehat{G}}^{0}\left(x^{k}\right)=A_{\widehat{G}}^{0}(x)$.

If $j \in J_{i}(x)$ and $J_{-i}(x)=\{\varnothing\}$ for some $i, j \in\{1,2\}$, then $\widehat{u}_{i}(x)=$ $l_{1}^{j}(x)+l_{2}^{j}(x)-u_{-i}(x), \widehat{u}_{-i}(x)=u_{-i}(x)$, and, therefore, $A_{\widehat{G}}^{0}(x)=l_{1}^{j}(x)+l_{2}^{j}(x)$. If $J_{-i}\left(x^{k}\right)=\{\varnothing\}$, then $\widehat{u}_{-i}\left(x^{k}\right)=u_{-i}\left(x^{k}\right)$ and $A_{\widehat{G}}^{0}\left(x^{k}\right)=l_{1}^{j}\left(x^{k}\right)+l_{2}^{j}\left(x^{k}\right)$. If $-j \in$ $J_{-i}\left(x^{k}\right)$, then $A_{\widehat{G}}^{0}\left(x^{k}\right)=\sum_{t \in\{1,2\}}\left(l_{1}^{t}\left(x^{k}\right)+l_{2}^{t}\left(x^{k}\right)\right)-u_{1}\left(x^{k}\right)-u_{2}\left(x^{k}\right)$. The set $J_{-i}(x)$ is empty if either $u_{-i}(x) \geq l_{-i}^{-j}(x)$ or $l_{-i}^{-j}(x)-u_{-i}(x) \leq u_{i}(x)-l_{i}^{-j}(x)$. Since $u_{i}(x) \geq l_{i}^{-j}(x)$ by (ii), we have, in both cases, that $l_{i}^{-j}(x)+l_{-i}^{-j}(x) \leq$ $u_{i}(x)+u_{-i}(x)$. Therefore, $\sum_{t \in\{1,2\}}\left(l_{1}^{t}(x)+l_{2}^{t}(x)\right)-u_{1}(x)-u_{2}(x) \leq l_{1}^{j}(x)+l_{2}^{j}(x)$. Thus, $\lim _{k \rightarrow \infty} A_{\widehat{G}}^{0}\left(x^{k}\right) \leq A_{\widehat{G}}^{0}(x)$. 
Case 2. Let $x \in \operatorname{rbd} S_{D}$. First notice that, since $x \notin S_{D}$, we have that $\widehat{u}_{i}(x)=u_{i}(x)$ for each $i$ and $A_{G}^{0}(x) \geq \max _{j \in\{1,2\}}\left\{l_{1}^{j}(x)+l_{2}^{j}(x)\right\}$. Obviously, if $x^{k} \in X \backslash S_{D}$ for each $k$, then $\lim _{k \rightarrow \infty} A_{\widehat{G}}^{0}\left(x^{k}\right)=\lim _{k \rightarrow \infty} A_{G}^{0}\left(x^{k}\right) \leq A_{G}^{0}(x)=$ $A_{\widehat{G}}^{0}(x)$. Consider a sequence $\left\{x^{k}\right\}$ lying in $S_{D}$.

If there are $i, j \in\{1,2\}$ such that $j \in J_{i}\left(x^{k}\right)$ and $J_{-i}\left(x^{k}\right)=\{\varnothing\}$ for infinitely many $k$, then, for such $k$ 's, $A_{\widehat{G}}^{0}\left(x^{k}\right)=l_{1}^{j}\left(x^{k}\right)+l_{2}^{j}\left(x^{k}\right)$, and, therefore, $\lim _{k \rightarrow \infty} A_{\widehat{G}}^{0}\left(x^{k}\right) \leq A_{\widehat{G}}^{0}(x)$. If there are $i, j \in\{1,2\}$ such that $j \in J_{i}\left(x^{k}\right)$ and $-j \in J_{-i}\left(x^{k}\right)$ for infinitely many $k$ 's, then, for such $k$ 's, $A_{\widehat{G}}^{0}\left(x^{k}\right)=\sum_{t \in\{1,2\}}\left(l_{1}^{t}\left(x^{k}\right)+l_{2}^{t}\left(x^{k}\right)\right)-u_{1}\left(x^{k}\right)-u_{2}\left(x^{k}\right)$. Since $u_{1}(x)+u_{2}(x) \geq$ $\max _{t \in\{1,2\}}\left\{l_{1}^{t}(x)+l_{2}^{t}(x)\right\}$ and the restriction of $A_{G}^{0}$ to $S$ is continuous, we have that $\lim _{k \rightarrow \infty} A_{\widehat{G}}^{0}\left(x^{k}\right) \leq \min _{t \in\{1,2\}}\left\{l_{1}^{t}(x)+l_{2}^{t}(x)\right\} \leq A_{G}^{0}(x)=A_{\widehat{G}}^{0}(x)$. Thus, we have shown that $A_{\widehat{G}}^{0}$ is upper semicontinuous on $X$.

By Lemma $7, \widehat{G}$ has a mixed strategy Nash equilibrium, $\widehat{\mu}=\left(\widehat{\mu}_{1}, \widehat{\mu}_{2}\right)$.

Let us show that $\widehat{\mu}\left(S_{D}\right)=0$. Assume, by way of contradiction, that $\widehat{\mu}\left(S_{D}\right)>0$. It is not difficult to see that $\widehat{\mu}\left(S_{D}\right)>0$ iff $\widehat{\mu}(x)>0$ for some $x=(z, z) \in S_{D}$ (see the Appendix, Lemma 10). Then $\widehat{\mu}_{i}(z)>0$ for each $i \in\{1,2\}$. By (iii), there exist $i, j \in\{1,2\}$ and some sequence $\left\{\left(x_{i}^{k}, z\right)\right\} \subset S^{j}$ converging to $x$ such that $\lim _{n \rightarrow \infty} l_{i}^{j}\left(x_{i}^{k}, z\right)>\widehat{u}_{i}(x)$. Let, without loss of generality, $j=-i$. By Lemma $8, \widehat{u}_{i}$ is strongly uniformly hospitable at $z$. That is, for every $\varepsilon>0$, there exists $\delta(\varepsilon)>0$ such that $z+\delta(\varepsilon) \in[0,1]$ and, for every $d_{i} \in(z, z+\delta(\varepsilon)), \widehat{u}_{i}\left(d_{i}, x_{-i}\right)>\widehat{u}_{i}\left(z, x_{-i}\right)-\varepsilon$ for all $x_{-i} \in X_{-i}$. It is worth noticing that $\delta(\varepsilon)$ is positive since $j=-i$.

Since $\widehat{\mu}_{i}(z)>0, \widehat{U}_{i}\left(\delta_{z}, \widehat{\mu}_{-i}\right)=\max _{\mu_{i} \in \Delta\left(X_{i}\right)} \widehat{U}_{i}\left(\mu_{i}, \widehat{\mu}_{-i}\right)$. On the other hand, $\widehat{U}_{i}\left(\delta_{z}, \widehat{\mu}_{-i}\right)=\widehat{\mu}_{-i}(z) u_{i}(z, z)+\int_{[0,1] \backslash\{z\}} \widehat{u}_{i}\left(z, x_{-i}\right) d \widehat{\mu}_{-i}$. Fix some $\varepsilon \in$ $\left(0, \frac{1}{2} \widehat{\mu}_{-i}(z)\left(l_{i}^{-i}(z, z)-\widehat{u}_{i}(z, z)\right)\right)$. Pick some $d_{i} \in(z, z+\delta(\varepsilon))$ such that $\widehat{u}_{i}\left(d_{i}, z\right)=l_{i}^{-i}\left(d_{i}, z\right)>\frac{1}{2}\left(l_{i}^{-i}(z, z)+\widehat{u}_{i}(z, z)\right)$. Then $\widehat{U}_{i}\left(\delta_{d_{i}}, \widehat{\mu}_{-i}\right)-\widehat{U}_{i}\left(\delta_{z}, \widehat{\mu}_{-i}\right)>$ $\widehat{\mu}_{-i}(z)\left(\widehat{u}_{i}\left(d_{i}, z\right)-\widehat{u}_{i}(z, z)\right)-\varepsilon>0$, a contradiction.

Therefore, $\widehat{U}_{i}(\widehat{\mu})=U_{i}(\widehat{\mu}), i=1,2$. Since $\widehat{\mu}$ is a mixed strategy equilibrium of $\widehat{G}$ and, by construction, $\widehat{U}_{i}(\mu) \geq U_{i}(\mu)$ for each $i \in\{1,2\}$ and every $\mu \in \triangle(X)$, we conclude that $\widehat{\mu}$ is also a mixed strategy Nash equilibrium of $G$.

Remark Since $\widehat{u}_{1}(x)+\widehat{u}_{2}(x)=\sum_{t \in\{1,2\}}\left(l_{1}^{t}(x)+l_{2}^{t}(x)\right)-u_{1}(x)-u_{2}(x)$ in some cases, assuming that the restriction of $A_{G}^{0}$ to $S$ is only upper semicontinuous would not be sufficient for the purposes of Theorem 3 even if its assumption (ii) were replaced with the stronger assumption (iv) of Theorem 
5.1 of Bagh (2010). On the other hand, it is useful to notice that the restrictions of $\widehat{u}_{1}$ and $\widehat{u}_{2}$ to $S$ need not be reciprocally upper semicontinuous (see Bagh 2010, p. 1265, footnote 24, for the definition) if the restrictions of $u_{1}$ and $u_{2}$ to $S$ are reciprocally upper semicontinuous.

Example 4 demonstrates that Theorem 3 can be used in place of Theorem $5 \mathrm{~b}$ to replicate the equilibrium existence results obtained by Ball (1999).

Example 4 Consider the following spatial voting model (see Ball, 1999, Example 1). Two candidates are competing in an election for public office. The electorate is distributed uniformly along the ideological spectrum $[0,1]$. During the electoral campaign, each candidate $i$ announces, simultaneously with the other candidate, a platform, denoted by $x_{i}$. The probability $P_{i}\left(x_{i}, x_{-i}\right)$ that candidate $i$ candidate wins the election is defined as follows:

$$
P_{i}\left(x_{i}, x_{-i}\right)=\left\{\begin{array}{l}
\frac{x_{i}+x_{-i}}{2} \text { for } 0 \leq x_{i}<x_{-i} \leq 1, \\
\frac{1}{2} \text { for } 0 \leq x_{i}=x_{-i} \leq 1 \\
1-\frac{x_{i}+x_{-i}}{2} \text { for } 0 \leq x_{-i}<x_{i} \leq 1 .
\end{array}\right.
$$

As in Ball (1999, p. 541), candidates 1 and 2's policy preferences on $[0,1]$ are represented by $h_{1}(z)=-\frac{1}{2}(z-1)^{2}$ and $h_{2}(z)=-\frac{1}{2} z^{2}$. The candidates are assumed to be office-motivated. Let the candidates' office motivation parameters be $k_{1}=.05$ and $k_{2}=3$, respectively. Then candidate $i$ 's payoff function is

$$
u_{i}\left(x_{i}, x_{-i}\right)=P_{i}\left(x_{i}, x_{-i}\right)\left(h_{i}\left(x_{i}\right)+k_{i}\right)+\left(1-P_{i}\left(x_{i}, x_{-i}\right)\right) h_{i}\left(x_{-i}\right) .
$$

The game has no pure strategy Nash equilibrium.

It is strongly uniformly hospitable since $S_{D}=S \backslash\left\{\left(\frac{1}{2}, \frac{1}{2}\right)\right\}$ and the payoff functions are continuous at $\left(\frac{1}{2}, \frac{1}{2}\right)$. Obviously, the restriction of $u_{1}+u_{2}$ to the main diagonal of the unit square is a continuous function. By Theorem ??, the game has a mixed strategy Nash equilibrium.

\section{Appendix}

\section{The Ky Fan Minimax Inequality}

In Theorem 1 we use the Ky Fan minimax inequality in the following, slightly generalized form. (see, e.g., Ding and Park, 2002, Theorem 2.4; Lan and Wu, 2002, Theorem 3.4 for more general results). 
Lemma 9 Let $X$ be a compact convex set in a Hausdorff topological vector space, and let $f: X \times X \rightarrow \mathbb{R}$ satisfy:

(i) $f(x, x) \leq 0$ for each $x \in X$;

(i) $f(\cdot, y)$ is quasiconcave for each $y \in X$,

(ii) $f$ is 0-transfer lower semicontinuous in $y$.

Then there exists $\bar{y} \in X$ such that $f(x, \bar{y}) \leq 0$ for all $x \in X$.

Lemma 9 can be shown in a number of ways. Its conventional proofs are based either on the KKM lemma or on Browder's fixed point theorem, which are two equivalent results (see, for an in-depth discussion, Yannelis 1991). Let us give an outline of the proof using Browder's fixed point theorem. It proceeds by assuming, to the contrary, that, for each $y \in X$, there exists $x \in X$ such that $f(x, y)>0$. Then the correspondence $M: X \rightarrow X$ defined by $M(y)=\{x \in X: f(x, y)>0\}$ has nonempty values. The quasiconcavity of $f$ in $x$ implies that $M$ has convex values. Since $f$ is 0 transfer lower semicontinuous in $y, M$ has a multivalued selection with open lower sections (see, e.g., Prokopovych, 2011 for details), denoted by $M_{0}$ : $X \rightarrow X$. Then, by Lemma 5.1 of Yannelis and Prabhakar (1983), the convexvalued correspondence $\bar{M}_{0}: X \rightarrow X$ defined by $\bar{M}_{0}(x)=\operatorname{co} M_{0}(x)$ also has open lower sections. Therefore, by Browder's fixed point theorem, the selection has a fixed point, which contradicts (i).

\section{Proof of Theorem 2}

Suppose $\mu=\left(\mu_{1}, \ldots, \mu_{n}\right) \in \triangle(X) \backslash E_{\Gamma}$. Then there exists $\sigma \in \triangle(X)$ such that $F_{\Gamma}(\sigma, \mu)>0$. Since $\sigma$ is a vector of probability measures, there exists $d=\left(d_{1}, \ldots, d_{n}\right) \in X$ such that $F_{\Gamma}\left(\delta_{d}, \mu\right)>0$, where $\delta_{d}=\left(\delta_{d_{1}}, \ldots, \delta_{d_{n}}\right)$ is the vector of Dirac measures concentrated at $d_{1}, \ldots, d_{n}$. With some abuse of notation, we will write $F_{\Gamma}(d, \mu)$ in place of $F_{\Gamma}\left(\delta_{d}, \mu\right)$. Put $\varepsilon^{*}=F_{\Gamma}(d, \mu)$ and denote $\liminf _{w \rightarrow x} F_{G}(d, w)$ by $\underline{F}_{G}(d, x)$. Since $G$ is uniformly diagonally secure, there is $\bar{d} \in X$ such that $\underline{F}_{G}(\bar{d}, x)>F_{G}(d, x)-\frac{\varepsilon^{*}}{2}$ for all $x \in X$. Therefore $\underline{F}_{\Gamma}(\bar{d}, \mu)>F_{\Gamma}(d, \mu)-\frac{\varepsilon^{*}}{2}$, where $\underline{F}_{\Gamma}(\bar{d}, \mu)=\int_{X} \underline{F}_{G}(\bar{d}, x) d \mu$.

The lower semicontinuity of $\underline{F}_{G}$ in $x$ implies that $\underline{F}_{\Gamma}(\bar{d}, \cdot): \triangle(X) \rightarrow \mathbb{R}$ is lower semicontinuous (see Aliprantis and Border, 2006, Theorem 15.5). Consequently, $\underline{F}_{\Gamma}\left(\bar{d}, \mu^{\prime}\right)>F_{\Gamma}(d, \mu)-\frac{\varepsilon^{*}}{2}>0$ for all $\mu^{\prime}$ in some open neighborhood $\mathcal{N}_{\triangle(X)}(\mu)$ of $\mu$. Since $F_{G}(\bar{d}, x) \geq \underline{F}_{G}(\bar{d}, x)$ for all $x \in X$, we conclude that $F_{\Gamma}\left(\bar{d}, \mu^{\prime}\right) \geq \underline{F}_{\Gamma}\left(\bar{d}, \mu^{\prime}\right)>0$ for all $\mu^{\prime} \in \mathcal{N}_{\triangle(X)}(\mu)$, which means that $\Gamma$ is diagonally transfer continuous. 


\section{Proof of Lemma 5}

We have to show that for each $i \in I$, every $x_{i} \in X_{i}$, and every $\varepsilon>0$, there is a hospitable strategy $\sigma_{i} \in \triangle\left(X_{i}\right)$ such that $U_{i}\left(\sigma_{i}, x_{-i}\right)>u_{i}\left(x_{i}, x_{-i}\right)-\varepsilon$ for every $x_{-i} \in X_{-i}$.

Fix some $i \in I, x_{i} \in X_{i}$, and $\varepsilon>0$. Let $\sigma_{i}$ denote the uniform probability measure on $B_{i}^{+}\left(x_{i}, \varepsilon\right)$. By definition, $\sigma_{i}(C)=\frac{\lambda_{i}(C)}{\lambda_{i}\left(B_{i}^{+}\left(x_{i}, \varepsilon\right)\right)}$ for every Borel subset $C$ of $B_{i}^{+}\left(x_{i}, \varepsilon\right)$, therefore, $\sigma_{i}$ is absolutely continuous with respect to $\lambda_{i}$. Since $U_{i}\left(\sigma_{i}, x_{-i}\right)>u_{i}\left(x_{i}, x_{-i}\right)-\varepsilon$ for every $x_{-i} \in X_{-i}$, the only fact left to be proven is that $\sigma_{i}$ is a hospitable strategy.

Consider some $x_{-i} \in X_{-i}$ and a sequence of $\left\{x_{-i}^{k}\right\}, x_{-i}^{k} \in X_{-i}$, converging to it. Then $u_{i}\left(x_{i}, x_{-i}^{k}\right)$ tends to $u_{i}\left(x_{i}, x_{-i}\right)$ for $\lambda_{i}$-almost every $x_{i} \in X_{i}$. By the absolute continuity of $\sigma_{i}$, we conclude that $u_{i}\left(x_{i}, x_{-i}^{k}\right)$ converges to $u_{i}\left(x_{i}, x_{-i}\right)$ for $\sigma_{i}$-almost every $x_{i} \in X_{i}$. Then, by Lebesgue's dominated convergence theorem, $U_{i}\left(\sigma_{i}, x_{-i}^{k}\right)$ tends to $U_{i}\left(\sigma_{i}, x_{-i}\right)$.

\section{An Auxiliary Lemma}

Lemma 10 Let $X=[0,1] \times[0,1]$, and $\mu_{i} \in \triangle([0,1]), i=1,2$, and let $D$ be a Borel subset of $[0,1]$. Then the set $\bar{D}=\{(z, z) \in[0,1] \times[0,1]: z \in D\}$ has measure 0 for the product measure $\mu=\mu_{1} \times \mu_{2}$ iff $\mu(x)=0$ for every $x \in \bar{D}$.

Proof. Assume that $\mu(x)=0$ for every $x \in \bar{D}$. We have to show that $\mu(\bar{D})=0$.

First consider the case where, for some $i \in\{1,2\}, \mu_{i}(x)=0$ for all $x \in D$. Fix some arbitrary $\varepsilon>0$. Since $\mu_{i}$ has no atoms on $D$, there exists a finite disjoint collection $\left\{D_{k}\right\}_{k=1}^{m}$ of subsets of $D$ such that $D=\cup_{k=1}^{m} D_{k}$ and $\mu_{i}\left(D_{k}\right) \leq \varepsilon$. Then $\mu(\bar{D})=\sum_{k} \mu\left(D_{k} \times D_{k}\right)=\sum_{k} \mu_{i}\left(D_{k}\right) \mu_{-i}\left(D_{k}\right) \leq$ $\varepsilon \mu_{-i}(D) \leq \varepsilon$, which implies that $\mu(\bar{D})=0$.

Let both $\mu_{1}$ and $\mu_{2}$ have atoms on $D$. Each $\mu_{i}$, as a finite measure, can have only a countable number of atoms. Denote by $C_{i}$ the set of atoms of $\mu_{i}$ on $D$. Since $\mu(x)=0$ for every $x \in \bar{D}, C_{1} \cap C_{2}=\varnothing$.

Fix some arbitrary $\varepsilon>0$. Since $C_{1} \cap C_{2}=\varnothing$, it is possible to associate with every $x \in C_{1}$ a Borel set $D(x) \subset[0,1]$ containing $x$ such that $\mu_{1}(D(x)) \leq$ $2 \mu_{1}(x)$ and $\mu_{2}(D(x)) \leq \frac{\varepsilon}{2}$. Let $D_{1}=D \backslash C_{1}$. The set $D_{1}$ is Borel, and $\mu_{1}$ is nonatomic on it. Denote by $\bar{C}_{1}\left(\bar{D}_{1}\right)$ the set $\{(z, z) \in[0,1] \times[0,1]: z \in$ $\left.C_{1}\left(z \in D_{1}\right)\right\}$. It is clear that $\bar{C}_{1}$ is countable and $\mu\left(\bar{D}_{1}\right)=0$. Then, since 
$\bar{D}=\bar{C}_{1} \cup \bar{D}_{1}$ and $\bar{C}_{1} \cap \bar{D}_{1}=\varnothing$, we have that $\mu(\bar{D})=\mu\left(\bar{C}_{1}\right)+\mu\left(\bar{D}_{1}\right)=$ $\mu\left(\bar{C}_{1}\right) \leq \sum_{x \in C_{1}} \mu_{1}(D(x)) \mu_{2}(D(x)) \leq \sum_{x \in C_{1}} 2 \mu_{1}(x) \frac{\varepsilon}{2} \leq \varepsilon$.

\section{Example 1: Details}

Let us show that the game described in Example 1 with $V=2$ and $r=3$ is uniformly diagonally secure. Let $d \in X$ and, without loss of generality, $\varepsilon \in(0,1)$. Define $\bar{d}=\left(\bar{d}_{1}, \bar{d}_{2}\right)$ as follows:

$$
\bar{d}_{i}=\left\{\begin{array}{l}
d_{i} \text { if } d_{i}>0, \\
\frac{\varepsilon}{4} \text { if } d_{i}=0,
\end{array} \text { for } i=1,2 .\right.
$$

For any $x \in(0, V]$ denote by $y(x) \in(0, x)$ the root of the equation $\frac{x^{r}}{x^{r}+y^{r}} V=V-\frac{\varepsilon}{4}$. Such $y(x)$ exists and is unique since the function $f$ : $[0, x] \rightarrow \mathbb{R}$ defined by $f(y)=\frac{x^{r}}{x^{r}+y^{r}} V$ is strictly decreasing and continuous, and, moreover, $f(0)=V$ and $f(x)=\frac{V}{2}$. We will often use the fact that $y(x)<x$ for every $x \in(0, V]$.

There are a number of cases to consider.

Case 1. Let $d=(0,0)$. Then $\bar{d}_{1}=\bar{d}_{2}=\frac{\varepsilon}{4}$. We shall show that, for every $x \in X$, there exists a neighborhood $\mathcal{N}_{X}(x)$ of $x$ in $X$ such that $F_{G}(\bar{d}, w)>$ $F_{G}(d, x)-\varepsilon$ for all $w \in \mathcal{N}_{X}(x)$.

For $x=(0,0)$, put $\mathcal{N}_{X}(x)=B_{X}\left(x, \frac{\varepsilon}{4}\right)$. Since $F_{G}(d, x)=0$, we have to show that $F_{G}(\bar{d}, w)>-\varepsilon$ for all $w \in \mathcal{N}_{X}(x)$. $-\varepsilon$.

If $w=(0,0)$, then $F_{G}(\bar{d}, w)=A_{G}(\bar{d}, w)-A_{G}^{0}(w)=\left(V-\frac{\varepsilon}{2}\right)-\frac{V}{2}=\frac{V}{2}-\frac{\varepsilon}{2}>$

If $w=\left(w_{1}, 0\right)$ with $w_{1}>0$, then, since $w_{1}<\frac{\varepsilon}{4}, F_{G}(\bar{d}, w)>\left(V-\frac{\varepsilon}{2}\right)-$ $\left(\frac{V}{2}-w_{1}\right)>-\varepsilon$.

If $w=\left(w_{1}, w_{2}\right)$ with $w_{1} w_{2}>0$, then $F_{G}(\bar{d}, w)>\left(V-\frac{\varepsilon}{2}\right)-\left(V-w_{1}-w_{2}\right)>$ $-\varepsilon$.

Let $x=\left(x_{1}, 0\right)$ with $x_{1} \in(0, V]$. Then $F_{G}(d, x)=\frac{V}{4}-\left(\frac{V}{2}-x_{1}\right)$. Put $\mathcal{N}_{X}(x)=B_{X}\left(x, \frac{1}{4} \min \left\{x_{1}, y\left(\frac{\varepsilon}{4}\right)\right\}\right)$. Consider $w \in \mathcal{N}_{X}(x)$.

If $w=\left(w_{1}, 0\right)$, then $F_{G}(\bar{d}, w)>\left(\frac{V}{2}-\frac{\varepsilon}{4}-\frac{\varepsilon}{4}\right)-\left(\frac{V}{2}-w_{1}\right)$.

If $w=\left(w_{1}, w_{2}\right)$ with $w_{1} w_{2}>0$, then $F_{G}(\bar{d}, w)>\left(V-\frac{\varepsilon}{4}-\bar{d}_{1}-\bar{d}_{2}\right)-(V-$ $\left.w_{1}-w_{2}\right)$. It is clear that $F_{G}(\bar{d}, w)-F_{G}(d, x)>-\varepsilon$ for all $w \in \mathcal{N}_{X}(x)$.

For $x=\left(x_{1}, x_{2}\right) \in(0, V] \times(0, V], F_{G}(d, x)=-A_{G}^{0}(x)=-\left(V-x_{1}-\right.$ $\left.x_{2}\right)$. Put $\mathcal{N}_{X}(x)=B_{X}\left(x, \frac{1}{4} \min \left\{x_{1}, x_{2}, y\left(\frac{\varepsilon}{4}\right)\right\}\right)$. For $w \in \mathcal{N}_{X}(x)$ we have 
$F_{G}(\bar{d}, w)=A_{G}(\bar{d}, w)-\left(V-w_{1}-w_{2}\right)>\left(-\bar{d}_{1}-\bar{d}_{2}\right)-\left(V-w_{1}-w_{2}\right)$. Therefore, $F_{G}(\bar{d}, w)-F_{G}(d, x)>-\bar{d}_{1}-\bar{d}_{2}+w_{1}+w_{2}-x_{1}-x_{2}>-\varepsilon$.

Case 2. Let $d=\left(d_{1}, 0\right)$ with $d_{1} \in(0, V]$.

For $x=(0,0), F_{G}(d, x)=\left(\frac{V}{2}-d_{1}+\frac{V}{4}\right)-\frac{V}{2}=\frac{V}{4}-d_{1}$. Put $\mathcal{N}_{X}(x)=$ $B_{X}\left(x, \frac{1}{4} \min \left\{y\left(d_{1}\right), y\left(\frac{\varepsilon}{4}\right)\right\}\right)$ and consider $w \in \mathcal{N}_{X}(x)$.

If $w=(0,0)$, then $F_{G}(\bar{d}, w)=\left(V-d_{1}-\frac{\varepsilon}{4}\right)-\frac{V}{2}=\frac{V}{2}-d_{1}-\frac{\varepsilon}{4}$.

If $w=\left(w_{1}, 0\right)$ with $w_{1}>0$, then $F_{G}(\bar{d}, w)>\left(\frac{V}{2}-d_{1}+V-\frac{\varepsilon}{4}-\frac{\varepsilon}{4}\right)-\left(\frac{V}{2}-\right.$ $\left.w_{1}\right)=V-d_{1}-\frac{\varepsilon}{2}+w_{1}$.

If $w=\left(0, w_{2}\right)$ with $w_{2}>0$, then $F_{G}(\bar{d}, w)>\left(V-\frac{\varepsilon}{4}-d_{1}+\frac{V}{2}-\frac{\varepsilon}{4}\right)-\left(\frac{V}{2}-\right.$ $\left.w_{2}\right)=V-d_{1}-\frac{\varepsilon}{2}+w_{2}$.

If $w=\left(w_{1}, w_{2}\right)$ with $w_{1} w_{2}>0$, then $F_{G}(\bar{d}, w)>\left(V-\frac{\varepsilon}{4}-d_{1}+V-\frac{\varepsilon}{4}-\right.$ $\left.\frac{\varepsilon}{4}\right)-\left(V-w_{1}-w_{2}\right)=V-d_{1}-\frac{3 \varepsilon}{4}+w_{1}+w_{2}$.

Therefore, $F_{G}(\bar{d}, w)-F_{G}(d, x)>-\varepsilon$ for all $w \in \mathcal{N}_{X}(x)$.

For $x=\left(x_{1}, 0\right)$ with $x_{1} \in(0, V], F_{G}(d, x)=\left(\frac{V}{2}-d_{1}\right)-\left(\frac{V}{2}-x_{1}\right)$. Put $\mathcal{N}_{X}(x)=B_{X}\left(x, \frac{1}{4} \min \left\{\varepsilon, x_{1}, y\left(d_{1}\right)\right\}\right)$ and consider $w \in \mathcal{N}_{X}(x)$.

If $w=\left(w_{1}, 0\right)$, then $F_{G}(\bar{d}, w)>\left(\frac{V}{2}-d_{1}-\frac{\varepsilon}{4}\right)-\left(\frac{V}{2}-w_{1}\right)$.

If $w=\left(w_{1}, w_{2}\right)$ with $w_{1} w_{2}>0$, then $F_{G}(\bar{d}, w)>\left(V-\frac{\varepsilon}{4}-d_{1}-\frac{\varepsilon}{4}\right)-(V-$ $\left.w_{1}-w_{2}\right)$.

Therefore, $F_{G}(\bar{d}, w)-F_{G}(d, x)>-\varepsilon$ for all $w \in N_{X}(x)$.

For $x=\left(0, x_{2}\right)$ with $x_{2} \in(0, V], F_{G}(d, x)=\left(u_{1}\left(d_{1}, x_{2}\right)+\frac{V}{4}\right)-\left(\frac{V}{2}-\right.$ $\left.x_{2}\right)$. Since $u_{1}$ is continuous at $\left(d_{1}, x_{2}\right)$, there exists $0<\gamma<\frac{x_{2}}{2}$ such that $u_{1}\left(d_{1}, w_{2}\right)-u_{1}\left(d_{1}, x_{2}\right)>-\frac{\varepsilon}{4}$ for any $w_{2} \in\left(x_{2}-\gamma, x_{2}+\gamma\right) \cap[0, V]$. Put $\mathcal{N}_{X}(x)=B_{X}\left(x, \frac{1}{4} \min \left\{\gamma, y\left(\frac{\varepsilon}{4}\right)\right\}\right)$ and consider $w \in \mathcal{N}_{X}(x)$.

If $w=\left(0, w_{2}\right)$, then $F_{G}(\bar{d}, w)=\left(u_{1}\left(d_{1}, w_{2}\right)+\frac{V}{2}-\frac{\varepsilon}{4}\right)-\left(\frac{V}{2}-w_{2}\right)$.

If $w=\left(w_{1}, w_{2}\right)$ with $w_{1} w_{2}>0$, then $F_{G}(\bar{d}, w)>\left(u_{1}\left(d_{1}, w_{2}\right)+V-\frac{\varepsilon}{4}-\right.$ $\left.\frac{\varepsilon}{4}\right)-\left(V-w_{1}-w_{2}\right)$.

It is obvious that $F_{G}(\bar{d}, w)-F_{G}(d, x)>-\varepsilon$ for all $w \in \mathcal{N}_{X}(x)$.

For $x=\left(x_{1}, x_{2}\right)$ with $x_{1} x_{2}>0, F_{G}(d, x)=u_{1}\left(d_{1}, x_{2}\right)-\left(V-x_{1}-x_{2}\right)$. There exists $0<\gamma<\frac{x_{2}}{2}$ such that $u_{1}\left(d_{1}, w_{2}\right)-u_{1}\left(d_{1}, x_{2}\right)>-\frac{\varepsilon}{4}$ for any $w_{2} \in\left(x_{2}-\gamma, x_{2}+\gamma\right) \cap[0, V]$. Put $\mathcal{N}_{X}(x)=B_{X}\left(x, \frac{1}{4} \min \left\{\varepsilon, \gamma, x_{1}\right\}\right)$ and consider $w \in \mathcal{N}_{X}(x)$. Then $F_{G}(\bar{d}, w)>\left(u_{1}\left(d_{1}, w_{2}\right)-\frac{\varepsilon}{4}\right)-\left(V-w_{1}-w_{2}\right)$, and, therefore, $F_{G}(\bar{d}, w)-F_{G}(d, x)>-\varepsilon$.

Case 3. Let $d=\left(d_{1}, d_{2}\right) \in(0, V] \times(0, V]$. 
For $x=(0,0), F_{G}(d, x)=\left(V-d_{1}-d_{2}\right)-\frac{V}{2} . \operatorname{Put} \mathcal{N}_{X}(x)=B_{X}\left(x, \frac{1}{4} \min \left\{\varepsilon, y\left(d_{1}\right), y\left(d_{2}\right)\right\}\right)$ and consider $w \in \mathcal{N}_{X}(x)$.

If $w=(0,0)$, then $F_{G}(\bar{d}, w)=F_{G}(d, x)$.

If $w=\left(w_{1}, 0\right)$, then $F_{G}(\bar{d}, w)>\left(\frac{V}{2}-d_{1}+V-\frac{\varepsilon}{4}-d_{2}\right)-\left(\frac{V}{2}-w_{1}\right)$, and, therefore, $F_{G}(\bar{d}, w)-F_{G}(d, x)>-\varepsilon$.

If $w=\left(w_{1}, w_{2}\right)$ with $w_{1} w_{2}>0$, then $F_{G}(\bar{d}, w)>\left(V-\frac{\varepsilon}{4}-d_{1}+V-\frac{\varepsilon}{4}-\right.$ $\left.d_{2}\right)-\left(V-w_{1}-w_{2}\right)$.

Therefore, $F_{G}(\bar{d}, w)-F_{G}(d, x)>-\varepsilon$ for all $w \in \mathcal{N}_{X}(x)$.

For $x=\left(x_{1}, 0\right)$ with $x_{1} \in(0, V], F_{G}(d, x)=\left(\frac{V}{2}-d_{1}+u_{2}\left(x_{1}, d_{2}\right)\right)-\left(\frac{V}{2}-x_{1}\right)$. Pick $0<\gamma<\frac{x_{1}}{2}$ such that $u_{2}\left(w_{1}, d_{2}\right)-u_{2}\left(x_{1}, d_{2}\right)>-\frac{\varepsilon}{4}$ for any $w_{1} \in$ $\left(x_{1}-\gamma, x_{1}+\gamma\right) \cap[0, V]$. Put $\mathcal{N}_{X}(x)=B_{X}\left(x, \frac{1}{4} \min \left\{\varepsilon, \gamma, y\left(d_{1}\right)\right\}\right)$ and consider $w \in \mathcal{N}_{X}(x)$.

If $w=\left(w_{1}, 0\right)$, then $F_{G}(\bar{d}, w)>\left(\frac{V}{2}-d_{1}+u_{2}\left(w_{1}, d_{2}\right)\right)-\left(\frac{V}{2}-w_{1}\right)$.

If $w=\left(w_{1}, w_{2}\right)$ with $w_{1} w_{2}>0$, then $F_{G}(\bar{d}, w)>\left(V-\frac{\varepsilon}{4}-d_{1}+u_{2}\left(w_{1}, d_{2}\right)\right)-$ $\left(V-w_{1}-w_{2}\right)$.

Therefore, $F_{G}(\bar{d}, w)-F_{G}(d, x)>-\varepsilon$ for all $w \in \mathcal{N}_{X}(x)$.

For $x=\left(x_{1}, x_{2}\right) \in(0, V] \times(0, V], F_{G}(d, x)=u_{1}\left(d_{1}, x_{2}\right)+u_{2}\left(x_{1}, d_{2}\right)-$ $\left(V-x_{1}-x_{2}\right)$. Pick $0<\gamma<\frac{1}{2} \min \left\{x_{1}, x_{2}\right\}$ such that, for each $i \in\{1,2\}$, $u_{i}\left(d_{i}, w_{-i}\right)-u_{i}\left(d_{i}, x_{-i}\right)>-\frac{\varepsilon}{4}$ for all $w_{-i} \in\left(x_{-i}-\gamma, x_{-i}+\gamma\right) \cap[0, V]$. Put $\mathcal{N}_{X}(x)=B_{X}\left(x, \frac{1}{4} \min \{\varepsilon, \gamma\}\right)$. For $w=\left(w_{1}, w_{2}\right) \in \mathcal{N}_{X}(x)$ we have $F_{G}(\bar{d}, w)=$ $u_{1}\left(d_{1}, w_{2}\right)+u_{2}\left(w_{1}, d_{2}\right)-\left(V-w_{1}-w_{2}\right)$. Therefore, $F_{G}(\bar{d}, w)-F_{G}(d, x)>-\varepsilon$.

\section{References}

Aliprantis CD, Border KC (2006) Infinite Dimensional Analysis, A Hitchhiker's Guide, 3rd ed. Berlin: Springer-Verlag

Bagh A, Jofre A (2006) Reciprocal upper semicontinuity and better reply secure games: a comment. Econometrica 74:1715-1751

Bagh A (2010) Variational convergence: Approximation and existence of equilibria in discontinuous games. J Econ Theory 145:1244-1268

Ball R (1999) Discontinuity and nonexistence of equilibrium in the probabilistic spatial voting model. Soc Choice Welfare 16:533-555

Barelli P, Soza I (2009) On the existence of Nash equilibria in discontinuous and qualitative games. University of Rochester, Mimeo 
Baye MR, Tian G, Zhou J (1993) Characterization of the existence of equilibria in games with discontinuous and non-quasiconcave payoffs. Rev Econ Stud 60:935-948

Baye MR, Kovenock D, de Vries CG (1994) The solution to the Tullock rent-seeking game when $R>2$ : Mixed-strategy equilibria and mean dissipation rates. Public Choice 81:363-380

Baye MR, Kovenock D, de Vries CG (1996) The all-pay auction with complete information. Econ Theory 8:291-305

Bich P (2009) Existence of pure Nash equilibria in discontinuous and non quasiconcave games. Int J Game Theory 38:395-410

Carbonell-Nicolau O, Ok EA (2007) Voting over income taxation. J Econ Theory 134:249-286

Carmona G (2005) On the existence of equilibria in discontinuous games: three counterexamples. Int J Game Theory 33:181-187

Carmona G (2011) Understanding some recent existence results for discontinuous games. Econ Theory 48:31-45

de Castro LI (2011) Equilibria existence in regular discontinuous games. Econ Theory 48:67-85

Dasgupta P, Maskin E (1986) The existence of equilibrium in discontinuous economic games, I: Theory. Rev Econ Stud 53:1-26

Ding XP, Park JY (2002) Continuous selection theorem, coincidence theorem, and generalized equilibrium in $L$-convex spaces. Comput Math Appl 44:95-103

Duggan J (2007) Equilibrium existence for zero-sum games and spatial models of elections. Games and Econ Behav 60:52-74

Glycopantis D, Muir A (2004) The compactness of $\operatorname{Pr}(K)$. Adv Math Econ 6:39-53

Lan KQ, Wu JH (2002) A fixed-point theorem and applications to problems on sets with convex sections and to Nash equilibria. Math Comput Model 36:139-145

McLennan A, Monteiro PK, Tourky R (2011) Games with discontinuous payoffs: a strengthening of Reny's existence theorem. Econometrica 79:16431664

Monteiro PK, Page FH (2007) Uniform payoff security and Nash equilibrium in compact games. J Econ Theory 134:566-575

Page FH, Monteiro PK (2003) Three principles of competitive nonlinear pricing. J Math Econ 39:63-109 
Prokopovych P (2011) On equilibrium existence in payoff secure games. Econ Theory 48:5-16

Prokopovych P (2012) The single deviation property in games with discontinuous payoffs. Econ Theory. DOI 10.1007/s00199-012-0696-0

Reny PJ (1999) On the existence of pure and mixed strategy Nash equilibria in discontinuous games. Econometrica 67:1029-1056

Reny PJ (2009) Further results on the existence of Nash equilibria in discontinuous games. University of Chicago, mimeo

Reny PJ (2011) Strategic approximation of discontinuous games. Econ Theory 48:17-29

Simon L (1987) Games with discontinuous payoffs. Rev Econ Stud 54:569597

Sion M, and Wolfe P (1957) On a game without a value. In: Contributions to the Theory of Games, III. Princeton: Ann Math Stud 39:299-306

Tian G (1992) Generalizations of the FKKM theorem and the Ky Fan minimax Inequality, with applications to maximal elements, price equilibrium, and complementarity. J Math Anal Appl 170:457-471

Tullock G (1980) Efficient rent-seeking. In: J.M. Buchanan et al. (Eds) Toward a Theory of the Rent-Seeking Society. College Station: Texas A\&M University Press

Yannelis NC, Prabhakar ND (1983) Existence of maximal elements and equilibria in linear topological spaces. J Math Econ 12:233-245

Yannelis NC (1991) The core of an economy without ordered preferences. In: Khan,M.A., Yannelis, N.C. (eds.) Equilibrium Theory in Infinite Dimensional Spaces, Berlin: Springer 